\title{
?1
}

TI 2020-020/II

Tinbergen Institute Discussion Paper

\section{All symmetric equilibria in differential games with public goods}

Niko Jaakkola ${ }^{1}$

Florian Wagener ${ }^{2}$

${ }^{1}$ University of Bologna

2 University of Amsterdam 
Tinbergen Institute is the graduate school and research institute in economics of Erasmus University Rotterdam, the University of Amsterdam and Vrije Universiteit Amsterdam.

Contact: discussionpapers@tinbergen.nl

More TI discussion papers can be downloaded at https://www.tinbergen.nl

Tinbergen Institute has two locations:

Tinbergen Institute Amsterdam

Gustav Mahlerplein 117

1082 MS Amsterdam

The Netherlands

Tel.: +31(0)205984580

Tinbergen Institute Rotterdam

Burg. Oudlaan 50

3062 PA Rotterdam

The Netherlands

Tel.: +31(0)10408 8900 


\title{
All symmetric equilibria in differential games with public goods*
}

\author{
Niko Jaakkola†, Florian Wagener ${ }^{\ddagger}$
}

April 14, 2020

\begin{abstract}
We characterise the entire set of symmetric stationary Markov-perfect Nash equilibria (MPE) in a differential game of public good investment, using the canonical problem of climate change as an example. We provide a sufficient and necessary condition for MPE and show how the entire set of MPE is constructed. The equilibrium in continuous strategies, unique in our context, is Pareto-dominated by any other equilibrium. If a Paretoundominated steady state exists, it is sustained by trigger-like strategies, with deviations above and below the steady state leading to different responses. We extend the theory of differential games to deal with payoffs under discontinuous strategies. Our methods work under general functional forms.
\end{abstract}

\section{Introduction}

Tackling climate change; the harvest of common-access renewable resources; noncooperative management of ecological systems with non-convex dynamics; joint investment ventures between firms; provision of effort between members of a team to a common project. All of these are examples of the problem of dynamic provision of public goods. It is well-known that, in the absence of cooperation, such problems involve free-riding. However, it is less clear which non-cooperative outcomes we should expect to observe, as such problems often have multiple Nash equilibria. In such a context, equilibrium selection becomes an issue. It has been suggested that climate negotiations, for example, can be thought of as an equilibrium selection mechanism (Dockner and Long, 1993). ${ }^{1}$

\footnotetext{
${ }^{*}$ We thank seminar audiences at ifo Institute, the Swedish University of Agricultural Sciences and University of Bologna for helpful comments.

${ }^{\dagger}$ University of Bologna, Piazza Scaravilli 2, 40126 Bologna, Italy; CESifo. Email: niko.jaakkola@unibo.it

${ }^{\ddagger}$ Amsterdam School of Economics, University of Amsterdam; Tinbergen Institute; CESifo. Email: F.O.O.Wagener@uva.nl

${ }^{1}$ There are of course many other interpretations of climate negotiations; see, for example, Harstad (2012).
} 
In this paper, we fully characterise the set of equilibria in a differential game of public good investment. ${ }^{2}$ For purposes of exposition, we present our results using the canonical example of transboundary stock pollution. However, the insights and methods will apply to many other examples. ${ }^{3}$ We expect our core messages to hold in other dynamic public goods problems, including the ones mentioned above.

We make two substantive points, and two technical advances. As our first substantive point, we show that there is a very large set of symmetric Markov-perfect equilibria (MPE). We give necessary and sufficient conditions for MPE (Theorem 4.16). We then demonstrate how to systematically construct the entire set (Theorem 4.18). We impose the natural requirement that equilibrium strategies be defined on the entire state space. Our equilibria generate payoffs for all strategy profiles over the entire state space, an issue the extant literature on multiple equilibria in differential games has grappled with.

Our second substantive point relates to welfare-ranking the equilibria. With rare exceptions, all of the previous literature has focused on continuous equilibria. We show that, in a class of stock pollution games, the continuous equilibriumunique in this class - is (weakly) Pareto-dominated everywhere by any other equilibrium (Theorem 5.2). That is, out of the many (symmetric) equilibria which could be selected for this game, the equilibrium in continuous strategiesthe focus of most of the past literature - is the worst one to pick, irrespective of the initial state. This raises questions regarding the positive and normative value of this equilibrium. If the players understand the game, and are able to communicate, then one would expect to never observe this equilibrium being played.

The first of our technical contributions relates to the solution methods we use, and how they expand the scope of problems which are tractable. The most common method of tackling MPE in differential games is algebraic, based on the use of very particular functional specifications which allow the analyst to guess the form of the value function, and then obtain the coefficients required to make the necessary conditions hold. We use geometric techniques instead, in particular the theory of planar nonlinear dynamical systems, and are able to obtain results which apply to a much wider class of problems. The methods we use also allow for straightforward computation of equilibria. We thus substantially extend the scope of applied problems which economists can tackle. Our methods are likely to be suited to deriving further general claims about Markov-perfect equilibria

\footnotetext{
${ }^{2}$ Differential games are dynamic games in continuous time. They have been widely used in many fields of economics, in particular in environmental and resource economics (Long, 2011, 2012). Recent literature in macroeconomics has also embraced continuous-time methods very similar to those used to analyse differential games (Achdou et al., 2014, 2017; Nuño and Moll, 2018).

${ }^{3}$ Recent contributions to the theory of public good investment games include Battaglini et al. (2014). Georgiadis (2015) and Cvitanić and Georgiadis (2016) study common investment problems, but in contrast to our setup, the payoff is only obtained upon completion of the project.
} 
in differential games.

The second technical advance we make is to treat discontinuous strategies properly. The possible non-existence of well-defined classical solutions to the differential equation governing state dynamics is a well-known problem in differential games. ${ }^{4}$ The typical solution is to rule out strategy profiles which generate illdefined solutions. However, as this restriction constrains strategy profiles, rather than action sets, it implies that the set of strategies the players are effectively allowed to choose from depends on the strategies chosen by the other players. This is an awkward solution to the problem, as the natural assumption in game theory is that while action sets may depend on the state or on time, they are independent of the actions being simultaneously chosen by other players. The alternative is either to just ignore discontinuous strategies, or to ensure existence and uniqueness by imposing Lipschitz continuity on allowed strategies. Our results show that these routes are also problematic, given that the resulting equilibrium is Pareto-dominated by any discontinuous equilibrium.

There is a large literature on various applications of differential games. We expect our technical advances - the treatment of discontinuous strategies and the geometric methods we use - to be applicable to much of this literature. Thus, a thorough reassessment of the existing results in many of these applications is called for.

The present paper consolidates and clarifies a long literature on multiple equilibria in differential games with public goods. ${ }^{5}$ Tsutsui and Mino (1990) and Dockner and Long (1993) pointed out how such games have multiple steady states which can be supported by MPE, but almost all of these equilibria are defined only on a proper subset of the state space. This begs the question of how a player's payoffs and optimal strategy are to be determined at a boundary point of the domain of definition (a point reachable by a feasible deviation). Rowat (2007) builds on this literature, constructing globally defined strategies by piecing together separate sections of locally defined equilibrium strategies. However, he ignored the possibility of discontinuous strategies. Wirl (2007) shows how, for some utility specifications, multiple globally defined equilibria may exist in continuous strategies; but he, also, ignores discontinuous strategies. Unlike these contributions, in our setting the continuous equilibrium is unique. We show it is Pareto-dominated by any member of a large set of equilibria in discontinuous strategies.

All of the above contributions work in very specific functional frameworks: typically, either linear-quadratic; a combination of isoelastic utility and linear state dynamics; or linearity in controls or states (perhaps after convenient transformations), leading to bang-bang solutions or to state-independent interior controls (an example of bang-bang solutions in a strategic context is given by Ekeland

\footnotetext{
${ }^{4}$ The issue is discussed in popular textbooks (Başar and Olsder, 1999; Fudenberg and Tirole, 1991; Dockner et al., 2000).

${ }^{5}$ Seminal contributions on public good investment games include Levhari and Mirman (1980) and Fershtman and Nitzan (1991).
} 
et al., 2015). Dockner and Wagener (2014) is one exception, demonstrating in a quite general framework how to characterise equilibria using geometric methods. We develop these methods further. ${ }^{6}$ Our results confirm that the commonly used algebraic 'guess-and-verify' approach is problematic, as the equilibria it generates are (at least in our context) Pareto-dominated by all other equilibria.

The reason why the previous literature has to a large extent ignored discontinuous strategies is that such strategy profiles may fail to generate well-defined and unique classical solutions to the differential equation governing the dynamics of the state variable. The conventional solution has been to rule out such strategy profiles, but as alluded to above, this is somewhat unsatisfactory. ${ }^{7}$ Dockner and Wagener (2014) do consider discontinuous strategies, but their focus on equilibrium strategies which generate value functions that are traditional viscosity solutions of the Hamilton-Jacobi-Bellman (HJB) equation (Bardi and CapuzzoDolcetta, 2008) made them miss the equilibria that we focus on-including the equilibria which give rise to the most favourable steady states. We allow arbitrary discontinuous strategies. The implied value functions are also viscosity solutions, but as extended to situations with discontinuous dynamics (Barles et al., 2013, 2014). Our application of these recent results may be of independent technical interest to researchers working on continuous-time models. ${ }^{8}$

The most closely related results to the present paper are Dockner and Sorger (1996) and Sorger (1998), who consider renewable resource management. These papers show the existence of two different continua of equilibria, each with a single point of discontinuity. However, they impose an admissibility requirement on strategies, with its associated issues. ${ }^{9}$ The latter paper also explicitly muses on the question of whether there might exist even more equilibria, and on the problem of welfare ranking these equilibria. We show how the entire set of equilibria can be constructed in the related setting of stock pollution control, and provide an unequivocal result comparing the continuous equilibrium to any other equilibrium. Our methods also make clear how the various other equilibria can be ranked; in particular, for any initial state, determining the best possible equilibrium becomes obvious.

The key insight from our analysis for the problem of climate change mitigation is that Pareto-undominated strategies will feature discontinuous emission paths. The undominated equilibria feature steady states supported by strategies resem-

\footnotetext{
${ }^{6}$ There are other exceptions; e.g. Sorger (1998), on which we expand below.

${ }^{7}$ The relationship between differential games and dynamic decision theory is unclear, given that a differential game does not have an extensive-form representation (Alós-Ferrer and Ritzberger, 2008). Our methods allow for a substantial relaxation of the restrictions required to ensure that an interpretation as a normal-form game, at least, is unproblematic.

${ }^{8}$ The recent contribution of Akao et al. (2018) utilises Filippov solutions to discontinuous differential equations, like we do (Filippov, 1988). However, they do not adapt payoffs accordingly, meaning that many of their proposed equilibria are in fact prone to profitable deviations. They also limit their attention to the linear-quadratic framework. We define payoffs so that they are continuous in the strategies, and our geometric methods allow us to derive results which apply in general frameworks.

${ }^{9}$ We elaborate further below; see especially footnote 13 .
} 
bling trigger equilibria. Carbon emissions will be high until a target steady-state level of atmospheric carbon concentrations is reached. Once the steady state is reached, emissions are ramped down rapidly. In the steady state, deviations by a country polluting too much will not be 'cleaned up' by the other parties, deterring countries from such deviations in the first place. Indeed, the response to a deviation may even involve the climate drifting to a worse steady state, with a higher atmospheric concentration. This suggests international discussions on climate change mitigation could be structured around target stabilisation levels for atmospheric carbon concentrations; the target level being reached with a relatively high emission rate, until an eventual rapid decarbonisation. However, our model remains very stylised and further work needs to be conducted to investigate such climate agreements before drawing strong policy conclusions.

The equilibria we derive are reminiscent of the equilibria obtained by Dutta and Sundaram (1993). However, their equilibria are not robust to even a vanishing amount of noise. Moreover, as long as the players have sufficient leeway to control the system, such equilibria do not exist. Lack of controllability can also support equilibria in which the efficient steady is reached eventually, but inefficiently slowly (Battaglini et al., 2014). In contrast, the equilibria we derive survive even when individual players have a lot of freedom in terms of controlling the system.

The rest of the paper proceeds as follows. In Section 2, we set up the basic model. Section 3 develops local necessary conditions for MPE. We then focus on the climate example in Section 4, to derive further global necessary conditions as cleanly as possible, and give conditions characterising MPE and a constructive method to obtain the entire set. Section 5 discusses welfare comparisons between equilibria. Finally, Section 6 discusses our results with a view to a future research agenda.

\section{Model}

Time is continuous and runs to infinity: $t \in[0, \infty)$. There are $N$ identical players, indexed by $i \in I \equiv\{1, \ldots, N\}$. Player $i$ has access to a control variable $q_{i}(t):[0, \infty) \rightarrow \mathcal{Q} \subset \mathbb{R}$. We assume the control set $\mathcal{Q}$ is convex and compact. ${ }^{10}$

There is a scalar state variable $x(t):[0, \infty) \rightarrow \mathcal{X} \subset \mathbb{R}$. The state space $\mathcal{X}$ is open and convex. The players use Markovian strategies, conditioning their actions on the current state variable only:

Definition 2.1. The strategy space $\mathscr{S}$ is the set of functions $\varphi: \mathcal{X} \rightarrow \mathcal{Q}$ which are piecewise continuously and boundedly differentiable. A Markovian strategy of player $i$ is a function $\phi_{i} \in \mathscr{S}$. A strategy profile is an $N$-tuple of strategies $\phi \equiv\left(\phi_{1}, \ldots, \phi_{n}\right) \in \mathscr{S}^{N}$.

\footnotetext{
${ }^{10}$ We could allow multivariate controls as in Dockner and Wagener (2014); our results would follow, given additional assumptions along the way. Our key insights are best conveyed without such complications.
} 
A function $\varphi: \mathcal{X} \rightarrow \mathcal{Q}$ is piecewise continuously and boundedly differentiable if $\mathcal{X}$ is covered by a finite number of closed intervals with disjunct interiors, such that $\varphi$ is continuously differentiable on each interior and such that its derivative is bounded on the closure.

Thus, at all times, a player using a Markovian strategy sets its action as $q_{i}(t)=$ $\phi_{i}(x(t))$. It is well-known that a best response to Markovian strategies is also Markovian (Maskin and Tirole, 2001).

The state variable is a public good (or public bad), in that the players' control variables reflect their contributions to investing in or disinvesting from it. The state evolution depends additively on the current state, but not on calendar time: the differential equation governing the state evolution is

$$
\dot{x}(t)=f(x(t), Q(t)) \equiv Q(t)-g(x(t)),
$$

where $Q(t)=\sum_{i \in I} q_{i}(t)$. We assume that $g(\cdot)$ is continuously differentiable. Given a set of Markovian strategies $\phi(x)$, then, $\dot{x}(t)=F(x(t))=f(x(t), \Phi(x(t)))$ with $\Phi(x)=\sum_{i \in I} \phi_{i}(x) .{ }^{11}$ As the Markovian strategies are not required to be continuous, the right-hand side of differential equation (1) may have discontinuities so that the equation may not have classical solutions. To deal with these, we define for each $x \in \mathcal{X}$ the lateral limits

$$
F^{-}(x) \equiv \lim _{x^{\prime} \uparrow x} F\left(x^{\prime}\right), \quad F^{+}(x) \equiv \lim _{x^{\prime} \downarrow x} F\left(x^{\prime}\right) .
$$

We can now specify the notion of solution to the dynamics given by equation (1).

Definition 2.2. Given a strategy profile $\phi$ and an initial state $x(0)=x_{0}$, a Filippov solution to equation (1) is an absolutely continuous function $x(t)$ which satisfies, for almost all t, the differential inclusion

$$
\dot{x}(t) \in \overline{\mathrm{co}}\left(F^{-}(x(t)), F^{+}(x(t))\right),
$$

where $\overline{\mathrm{co}}$ denotes the convex hull.

Note that, for Filippov solutions (Filippov, 1988), pointwise deviations in the strategy do not affect the law of motion; in particular, the law of motion at any state $\tilde{x}$ is independent of $\phi(\tilde{x})$, instead depending only on the left and right limits of $\phi(x)$ as $x \rightarrow \tilde{x}$.

Proposition 2.3. If $x(t)$ is a Filippov solution to equation (1), there exists a measurable function $0 \leq \bar{\mu}(t) \leq 1$ such that

$$
\dot{x}(t)=\bar{\mu}(t) F^{-}(x(t))+(1-\bar{\mu}(t)) F^{+}(x(t))
$$

almost everywhere in $t$.

\footnotetext{
${ }^{11}$ For brevity, we omit notation to indicate the dependence of $F$ on $\phi$.
} 
We relegate all proofs to Appendix A.

Thus, the crucial difference to most of the literature on differential games is that we explicitly deal with cases in which strategy profiles would lead to chattering solutions. We thus allow the space of admissible strategy profiles to simply be the product set of individual strategy spaces, as is standard in game theory. ${ }^{12}$

The primitive of the payoffs is the flow felicity function:

Definition 2.4. The felicity function of player $i$ is $\tilde{u}_{i}\left(x, q_{i}\right)$. To restrict ourselves to symmetric situations, we assume $\tilde{u}_{i}=\tilde{u}_{j}, \forall i, j$. We assume $\partial \tilde{u}_{i} / \partial q_{i}>0$ and $\partial^{2} \tilde{u}_{i} / \partial q_{i}^{2}<0$.

We next describe the optimisation problem of player $i$, given the strategy profile $\phi_{-i}(x)=\left(\phi_{1}(x), \ldots, \phi_{i-1}(x), \phi_{i+1}(x), \ldots, \phi_{N}(x)\right)$ of the remaining players. As a strategy profile does not necessarily pin down a unique trajectory, to determine $i$ 's best response we need to consider the underlying dynamic optimisation problem. To do this, we need to introduce some additional notation to deal with the complications presented by discontinuous strategies: namely, to adapt our notion of payoffs to Filippov solutions, and to handle the possibility of a multiplicity of trajectories satisfying the dynamic equation (3).

Introduce a covering $\mathscr{X}_{-i}=\left\{\mathcal{X}_{j}\right\}_{j=0}^{J}$ of $\mathcal{X}$ adapted to the strategy profile $\phi_{-i}$ : each $\mathcal{X}_{j}$ is a closed interval in $\mathcal{X}$ with the intersection $\mathcal{X}_{j} \cap \mathcal{X}_{k}$ consisting of a single common boundary point if $j$ and $k$ differ by one, and being empty otherwise. Denote by $\mathcal{I}_{j}=\mathcal{X}_{j-1} \cap \mathcal{X}_{j}$ the interface between two adjacent regions.

To be adapted to the strategy profile, the covering must satisfy that the restriction of $\phi_{-i}$ to int $\mathcal{X}_{j}$ is continuously and boundedly differentiable for each $j$. Denote by $\phi_{-i, j}=\left(\phi_{1, j}, \ldots, \phi_{i-1, j}, \phi_{i+1, j}, \ldots, \phi_{N, j}\right)$ the extension of this restriction to $\mathcal{X}_{j}$. In this manner, we can cleanly deal with the lateral limits of $\phi_{-i}(x)$ at a discontinuity: if $x \in \mathcal{I}_{j}$, we have $\phi_{-i}^{-}(x)=\phi_{-i, j-1}(x)$ and $\phi_{-i}^{+}(x)=\phi_{-i, j}(x)$.

For the same reason, we introduce the local action $q_{i, j}(t)$ for player $i$ in region $\mathcal{X}_{j}$ as the action played when the state satisfies $x(t) \in \operatorname{int} \mathcal{X}_{j}$. If the state is in the interface point belonging to $\mathcal{X}_{j-1}$ and $\mathcal{X}_{j}$, that is if $x(t) \in \mathcal{I}_{j}$, the action of the player is the weighted average

$$
\mu_{i, j}(t) q_{i, j-1}(t)+\left(1-\mu_{i, j}(t)\right) q_{i, j}(t)
$$

of the two active actions, where $\mu_{i, j}(t)$ is the local weight.

\footnotetext{
${ }^{12}$ The literature on differential games typically rules out strategy profiles which lead to e.g. chattering solutions. The implication is that there could be outcomes such that the effectively available set of strategies for a player depends on the strategies chosen by the other players, which goes against the spirit of games with simultaneous moves. Klein and Rady (2011) impose payoffs of $-\infty$ for strategy profiles which do not generate classically defined trajectories for the state. Thus, while the set of admissible strategy profiles coincides with the product of the strategy sets, the payoffs are very discontinuous in the space of strategy profiles, a feature which is a technical artefact rather than following from the economic fundamentals of the model.
} 


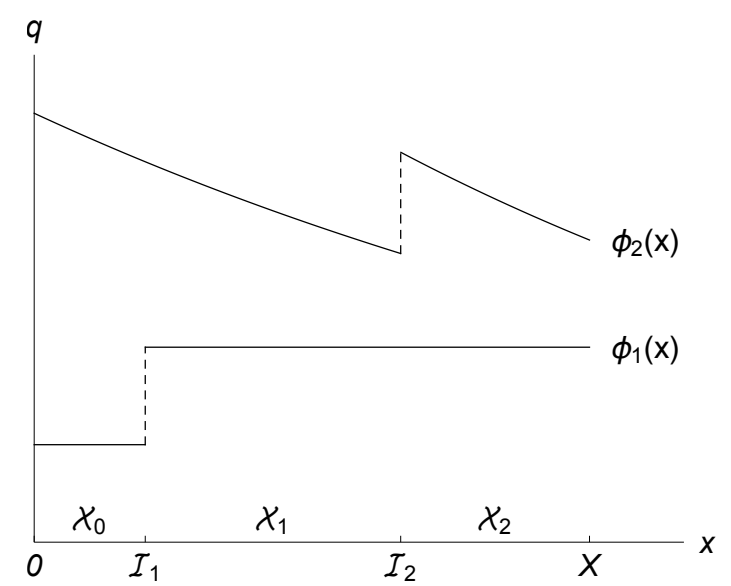

Figure 1: Covering adapted to strategies.

Figure 1 shows a three-player game with $\mathcal{X}=(0, X)$, two strategies with discontinuties, and a covering adapted to these. Player 3's action schedule is then

$$
\left(q_{3}(t), \mu_{3}(t)\right)=\left(q_{3,0}(t), q_{3,1}(t), q_{3,2}(t), \mu_{3,1}(t), \mu_{3,2}(t)\right) .
$$

Of course, at a given time, at most two of the $q_{3, j}(t)$ 's and at most one of the $\mu_{3, j}(t)$ 's is active.

More generally, the action schedule $\left(q_{i}, \mu_{i}\right)$ of player $i$ consists of the vector of local actions

$$
q_{i}(t)=\left(q_{i, 0}(t), \ldots, q_{i, J}(t)\right),
$$

with $q_{i, j}(t) \in \mathcal{Q}$ and the vector of local weights

$$
\mu_{i}(t)=\left(\mu_{i, 1}(t), \ldots, \mu_{i, J}(t)\right),
$$

with $\mu_{i, j}(t) \in[0,1]$, for all $t \geq 0$.

The local dynamics for player $i$ in the interior of $\mathcal{X}_{j}$ are given by

$$
\tilde{f}_{i, j}\left(x, q_{i, j}\right)=q_{i, j}+\Phi_{-i, j}(x)-g(x) \quad \text { if } x \in \operatorname{int} \mathcal{X}_{j},
$$

where $\Phi_{-i, j}(x)=\sum_{k \neq i} \phi_{k, j}(x)$, and on the interface $\mathcal{I}_{j}$ by

$$
\tilde{f}_{i, \mathcal{I}_{j}}\left(x, q_{i, j-1}, q_{i, j}, \mu_{i, j}\right)=\mu_{i, j} \tilde{f}_{i, j-1}\left(x, q_{i, j-1}\right)+\left(1-\mu_{i, j}\right) \tilde{f}_{i, j}\left(x, q_{i, j}\right) \quad \text { if } x \in \mathcal{I}_{j} .
$$

As the dynamics are discontinuous, actions do not necessarily result in unique state trajectories. A controlled trajectory $\left(x(t), q_{i}(t), \mu_{i}(t)\right)$ is possible, given a strategy profile $\phi_{-i}$ and the associated covering $\mathscr{X}_{-i}$, if for almost all $t$ we have

$$
\dot{x}(t)=f_{i}\left(x(t), q_{i}(t), \mu_{i}(t)\right) \equiv \tilde{f}_{i, j}\left(x, q_{i, j}\right), \quad \text { if } x(t) \in \operatorname{int} \mathcal{X}_{j},
$$


and

$$
\dot{x}(t)=f_{i}\left(x(t), q_{i}(t), \mu_{i}(t)\right) \equiv \tilde{f}_{i, \mathcal{I}_{j}}\left(x(t), q_{i}(t), \mu_{i}(t)\right), \quad \text { if } x(t) \in \mathcal{I}_{j} .
$$

We also define the covering $\mathscr{X}$ which is adapted to the full strategy profile $\phi$, exactly analogously to $\mathscr{X}_{-i}$ above. We have to make sure that, given strategies $\phi_{-i}$, a Markovian strategy $\phi_{i}(x)$ for player $i$ specifies a controlled trajectory $\left(x(t), q_{i}(t), \mu_{i}(t)\right)$. At points at which the strategy profile $\phi(x)$ is Lipschitz continuous (that is, in the interior of $\mathcal{X}_{j}$ for any $j$, so almost everywhere), the dynamics $F(x)=f(x, \Phi(x))$ are Lipschitz as well and the evolution of $x$ is uniquely defined by equation (3), the value of $\mu_{i}(t)$ being immaterial. Let $\tilde{x} \in \mathcal{I}_{j}$ be an interface point and let $\tilde{T}=\{t: x(t)=\tilde{x}\}$ be the set of times that $x(t)$ is at $\tilde{x}$. We have that $\dot{x}(t)=0$ almost everywhere on $\tilde{T}$, and for those times we have that $\tilde{\mu}_{i, j}(t)=\bar{\mu}_{i, j}$, where

$$
\bar{\mu}_{i, j}=\frac{F^{+}(\tilde{x})}{F^{+}(\tilde{x})-F^{-}(\tilde{x})}=\frac{\left|F^{+}(\tilde{x})\right|}{\left|F^{+}(\tilde{x})\right|+\left|F^{-}(\tilde{x})\right|} .
$$

Then $\left(q_{i}(t), \mu_{i}(t)\right)=\left(\phi_{i}(x(t)), \bar{\mu}_{i}\right)$ is an action schedule induced by $\phi_{i}(x)$. We call a controlled trajectory $\left(x(t), q_{i}(t), \mu_{i}(t)\right)$ associated with such an action schedule possible, given the full strategy profile $\phi$ and the covering $\mathscr{X}$ associated with $\phi$. A strategy profile may map to a non-singleton set of action schedules. We will shortly, when defining utility, pick out the subset of these (possibly a unique action schedule) which maximise utility. We therefore do not have to extend our strategy space: in the case of multiple possible controlled trajectories, we assume the existence of a mechanism that picks out a unique utility maximising action schedule.

We need to adapt the felicity function to the Filippov solution we have used to define the dynamics of the state, as follows:

Definition 2.5. The flow payoff of player $i$ is

$$
u_{i}\left(x, q_{i}, \mu_{i}\right)=\tilde{u}_{i}\left(x, q_{i, j}\right) \quad \text { if } x \in \operatorname{int} \mathcal{X}_{j},
$$

and

$$
u_{i}\left(x, q_{i}, \mu_{i}\right)=\mu_{j} \tilde{u}_{i}\left(x, q_{i, j-1}\right)+\left(1-\mu_{j}\right) \tilde{u}_{i}\left(x, q_{i, j}\right) \quad \text { if } x \in \mathcal{I}_{j} .
$$

We will discuss the flow payoffs momentarily.

It is useful to first introduce the overall payoffs from the game. Both players discount future payoffs at a common rate $\rho \in(0, \infty)$. Then:

Definition 2.6. Given a strategy profile $\phi_{-i}(x)$ and an initial state $x_{0}$, the utility of a strategy $\phi_{i}(x)$ to a player $i$ is

$$
J^{i}\left(x_{0} ;\left(\phi_{i}(x), \phi_{-i}(x)\right)\right)=\sup _{\mathcal{T}_{x_{0}, \phi, \mathscr{X}}} \int_{0}^{\infty} \exp (-\rho t) u_{i}\left(x, q_{i}, \mu_{i}\right) \mathrm{d} t,
$$


where $\mathcal{T}_{x_{0}, \phi, \mathscr{X}}$ is the set of all possible trajectories for $\left(x, q_{i}, \mu_{i}\right)$ with initial state $x_{0}$, given the strategy profile $\phi$ and the associated covering $\mathscr{X}$.

The value of the profile $\phi_{-i}(x)$ to player $i$ is

$$
V^{i}\left(x_{0} ; \phi_{-i}(x)\right)=\sup _{\mathcal{T}_{x_{0}, \phi_{-i}, \mathscr{X}_{-i}}} \int_{0}^{\infty} \exp (-\rho t) u_{i}\left(x, q_{i}, \mu_{i}\right) \mathrm{d} t
$$

where $\mathcal{T}_{x_{0}, \phi_{-i}, \mathscr{X}_{-i}}$ is the set of all possible trajectories with initial state $x_{0}$, given the strategy profile $\phi_{-i}$ and the associated covering $\mathscr{X}_{-i}$.

A strategy $\phi_{i}(x)$ is a best response to a strategy profile $\phi_{-i}(x)$, if

$$
V^{i}\left(x_{0} ; \phi_{-i}(x)\right)=J\left(x_{0} ;\left(\phi_{i}(x), \phi_{-i}(x)\right)\right) \text {. }
$$

Note that the utility of a strategy and the value of the profile are well-defined quantities, even if a supremum is taken over several possible trajectories. When actually playing the game, a coordination problem may arise, as different players may choose different action schedules that are consistent with the same strategy $\phi_{i}$. We take here advantage of the fact that we focus on symmetric strategies and posit that the players coordinate on choosing identical action schedules, which enables them to actually realise the value of the associated strategy.

Let us discuss the payoffs specified in Definition 2.5 given a strategy profile $\phi(x)$. The first thing to note about the flow payoff is that, at every non-interface point $x$, i.e. where the strategies are continuous, the flow payoff is just the felicity flow $\tilde{u}_{i}\left(x, \phi_{i}(x)\right)$. Furthermore, where strategies are discontinuous but $\operatorname{sign}\left(F^{-}\right)=\operatorname{sign}\left(F^{+}\right)$, the dynamics move the system past the point of discontinuity in an instant of time of measure zero, so that this discontinuity has no impact on utility as given by equation (6). In these cases, equation (1) has a unique classically defined solution. In other words, for classically admissible strategies, our definition of Filippov solutions and accordingly adapted payoffs give exactly the outcome which the previous literature has used.

Our definition of the dynamics, however, also admits discontinuities. Consider first strategies such that there is a discontinuity, say at point $x=\tilde{x}$, so that $F^{-}(\tilde{x})>0>F^{+}(\tilde{x})$. In these cases, the system is being driven towards the point $\tilde{x}$, and the possibility of chattering trajectories arises. ${ }^{13}$ The payoffs we define are a weighted sum of the left and right limits of the payoffs, with the weights given by the force with which the dynamics push the system towards $x=\tilde{x}$. The specification captures the following notion: the system never sits still exactly at $x=\tilde{x}$; instead, it chatters between the two sides. If the force pushing the system to the right is much stronger than the force pushing back to the left,

\footnotetext{
${ }^{13}$ With classical solutions, actual chattering would only occur if also $F(\phi(\tilde{x}), \tilde{x}) \neq 0$. In the case $F(\phi(\tilde{x}), \tilde{x})=0$, however, trajectories (and hence payoffs) would not be well-defined for small deviations in strategies at point $x=\tilde{x}$. Hence such deviations could not be evaluated. The usual admissibility requirement would simply rule out such deviations, thus limiting a player's action set in a way which depended on the other players' actions, purely due to a technical requirement.
} 
then the system spends more time on the right side of $x=\tilde{x}$ and consequently the payoff reflects that. This outcome would result if, for example, time were partitioned into infinitesimal intervals during which the control variables were held constant at the value specified by the strategy for the state at the first moment of each interval (a so-called 'sample-and-hold' solution; see Clarke, 2004). Our notion of payoffs is also closely linked to the literature on optimal control over multidomains (Barles et al., 2013). However, in our case, the division of the state space into multiple domains is endogenous to the equilibrium, instead of being given as a primitive of the problem.

A second type of discontinuity we want to highlight occurs at a point $x=\tilde{x}$ such that $F^{-}(\tilde{x})<0<F^{+}(\tilde{x})$. In such situations, stabilising the dynamics at $\tilde{x}$ corresponds to the 'singular dynamics' case of Barles et al. (2013). The following proposition shows that strict concavity of the felicity function in $q_{i}$ implies that this is never an optimal situation. ${ }^{14}$

Proposition 2.7. Let $\left(x(t), q_{i}(t), \mu_{i}(t)\right)$ be a possible controlled trajectory such that $x(t)=\tilde{x} \in \mathcal{I}_{j}$ for $t \in\left[0, t_{1}\right]$ and

$$
\tilde{f}_{i, j-1}\left(\tilde{x}, q_{i, j-1}\right)<0 \quad \text { and } \quad \tilde{f}_{i, j}\left(\tilde{x}, q_{i, j}\right)>0 .
$$

Then there is an action schedule $\left(\hat{q}_{i}, \hat{\mu}_{i}\right)$ that coincides with $\left(q_{i}, \mu_{i}\right)$ for all $t \in$ $\left[t_{1}, \infty\right)$ that has the following properties:

(a) the controlled trajectory $\left(x(t), \hat{q}_{i}(t), \hat{\mu}_{i}(t)\right)$ is also possible;

(b) for either $k=j-1$ or $k=j$, and for all $t \in\left[0, t_{1}\right]$, we have

$$
\tilde{f}_{i, k}\left(\tilde{x}, \hat{q}_{i, k}(t)\right)=0
$$

(c) for all $t \in\left[0, t_{1}\right]$, we have

$$
u_{i}\left(x(t), \hat{q}_{i}(t), \hat{\mu}(t)\right)>u_{i}\left(x(t), q_{i}(t), \mu(t)\right) .
$$

Thus, our specification of dynamic trajectories and payoffs nests the notion of utilities and equilibrium outcomes for strategy profiles commonly called 'admissible', while also allowing the consideration of classically inadmissible strategies. ${ }^{15}$ It turns out that this extension is very important for our main results.

Finally, we define the game and our equilibrium concept.

Definition 2.8. The tuple $\Gamma=(N, \mathcal{X}, \mathcal{Q}, g, u, \rho)$ defines a symmetric differential game.

\footnotetext{
${ }^{14}$ There are also potential discontinuities in which the dynamics equal zero on one side; these will be dealt with in the course of the analysis.

${ }^{15}$ Our specification does not allow for equilibria with isolated stationary points, with the dynamics pushing in the same direction at a strictly positive rate on both sides, as in Dutta and Sundaram (1993).
} 
Definition 2.9. A stationary Markov-perfect Nash equilibrium, or MPE, of symmetric differential game $\Gamma$ is a strategy profile $\phi \in \mathscr{S}^{N}$ such that, for any initial state $x_{0} \in \mathcal{X}$, player index $i \in I$, the strategy $\phi_{i}$ is a best response to $\phi_{-i}$.

An MPE is continuous if all $\phi_{i}$ are continuous; otherwise it is discontinuous. A symmetric MPE is an MPE of a symmetric differential game such that $\phi_{i}=\phi_{j}$ for all $i, j \in I$.

The definition is standard and requires that, starting from any state $x_{0}$ in the state domain, each player's strategy is a best response to the other players' strategies. We will from now on focus solely on symmetric MPE.

\section{Markov-perfect Nash equilibrium}

We develop basic tools to construct the set of symmetric, stationary Markovperfect equilibria. We consider equilibria in which the value function is continuous (even though the strategies are allowed to be discontinuous). ${ }^{16}$ In this section, we give local necessary conditions for MPE, which will apply to public goods games in general.

In the subsequent section, we will derive further global necessary conditions restricting the types of discontinuities equilibrium strategies can have. The global conditions will be specific to a particular context, so we will there restrict ourselves to an important subclass of problems. We then prove a sufficiency result that strategies which satisfy all necessary conditions, as well as a local controllability condition, form a symmetric MPE. We finally show how construct all such strategies with an arbitrary number of discontinuities. While the analysis in that section applies to our particular setting, other settings can be studied using a similar approach.

We first obtain necessary conditions for an MPE that is piecewise continuously and boundedly differentiable. When such an equilibrium strategy is differentiable, it has to satisfy the Hamilton-Jacobi-Bellman (HJB) equation (Bardi and Capuzzo-Dolcetta, 2008, Propositions 2.8 and 1.9): ${ }^{17}$

$$
\rho V^{i}\left(x ; \phi_{-i}\right)=H_{i}\left(x, V_{i}^{\prime} ; \phi_{-i}\right) \equiv \max _{q_{i}} P_{i}\left(x, q_{i}, V_{i}^{\prime} ; \phi_{-i}\right),
$$

where $P_{i}$ is the unmaximised Hamiltonian:

$$
P_{i}\left(x, q_{i}, p_{i} ; \phi_{-i}\right)=u\left(x, q_{i}\right)+p_{i} f\left(x, q_{i}+\Phi_{-i}(x)\right) .
$$

Note that we have left out the region index $j$ for the sake of readability. Using equation (1), an interior maximiser $\hat{q}_{i}$ of $q_{i} \mapsto P_{i}\left(x, q_{i}, p_{i}\right)$ must satisfy the

\footnotetext{
${ }^{16}$ Equilibria which generate discontinuous values remain work in progress. The key messages of the present paper remain essentially unaffected. Details are available on request.

${ }^{17}$ We denote derivatives of univariate functions by a prime, and partial derivatives by subscripts, where convenient.
} 
necessary condition

$$
\frac{\partial u}{\partial q_{i}}\left(x, \hat{q}_{i}\right)+p_{i}=0 .
$$

There are corresponding conditions for boundary maximisers. The assumed strict concavity of the felicity function in $q_{i}$ ensures that these conditions are also sufficient and allows us to write the optimal control as

$$
\hat{q}_{i}=z\left(x, V_{i}^{\prime}\right) .
$$

Further, as we focus on symmetric equilibria only, for which $V_{i}(x)=V(x)$ for all $i \in I$, we obtain the equilibrium action as a function of the state $x$ and the shadow price $V^{\prime}(x)$ :

$$
q^{*}=q^{*}\left(x, V^{\prime}\right) .
$$

Substituting back into the HJB equation, at points where $V$ is differentiable, we find the first necessary condition for the value function of a MPE. Summarising:

Proposition 3.1 (Game Hamilton-Jacobi-Bellman equation). Let $V$ be the value function of a symmetric MPE. If $V$ is differentiable at $x$, then

$$
\rho V(x)=G\left(x, V^{\prime}(x)\right),
$$

where the game Hamilton function is defined as

$$
G(x, p) \equiv u\left(x, q^{*}(x, p)\right)+p\left(N q^{*}(x, p)-g(x)\right) .
$$

Equation (11) is a nonlinear first-order differential equation in $V(x)$. To solve it, we differentiate both sides with respect to $x$, rearrange, and introduce $p(x) \equiv$ $V^{\prime}(x)$, to obtain the shadow price equation

$$
p^{\prime}(x)=-\frac{-\rho p(x)+u_{x}-p(x) g^{\prime}(x)+(N-1) p(x) q_{x}^{*}}{N q^{*}-g(x)+(N-1) p(x) q_{p}^{*}},
$$

in which $q^{*}$ and its partial derivatives are evaluated at $(x, p)$. Following the approach taken in the literature (e.g. Tsutsui and Mino, 1990; Dockner and Long, 1993; Bressan, 2009; Dockner and Wagener, 2014), we use this equality to construct the shadow price system, which is the dynamical system in variables $(\tilde{p}(s), \tilde{x}(s))$ given by

$$
\tilde{x}^{\prime}(s)=h_{1}^{*}(\tilde{x}(s), \tilde{p}(s)), \quad \tilde{p}^{\prime}(s)=h_{2}^{*}(\tilde{x}(s), \tilde{p}(s)),
$$

where, evaluating $q^{*}$ at $(x, p)$,

$$
\begin{aligned}
& h_{1}^{*}(x, p) \equiv N q^{*}-g(x)+(N-1) p q_{p}^{*}, \\
& h_{2}^{*}(x, p) \equiv \rho p-u_{x}-p g^{\prime}-(N-1) p q_{x}^{*} .
\end{aligned}
$$

A remark on notation is useful here. Except for the optimal action function 
$q^{*}(\cdot)$, and (in Section 4) its inverse $p^{*}(\cdot)$, the asterisk will be used to denote quantities in the shadow price system. We will later set up its counterpart, the action system, for which we use the corresponding variable and function labels, but without the asterisk.

The auxiliary variable $s$ is used purely to parameterise the system (13), and has no economic interpretation, except in the case of $N=1$ (see below). We have judiciously chosen the equations of motion to mirror the denominator and numerator, respectively, of the right-hand side of equation (12) substituting $\tilde{p}$ for $p(x)$, and $\tilde{x}$ for $x$, as the arguments of all functions. In this way the indeterminacies of the fraction on the right hand side of (12) correspond to the steady states of the system (13). It is crucial to note that solution trajectories of system (13) will satisfy equation (12) where $h_{1}^{*}(x, p) \neq 0$, as along these trajectories $\mathrm{d} p / \mathrm{d} x=\tilde{p}^{\prime}(s) / \tilde{x}^{\prime}(s)$. Having thus made clear that the solution curves $(\tilde{p}(s), \tilde{x}(s))$ to the system (13) describe pieces of potential candidate equilibrium strategies $p(x)$ to the game, we hereafter drop the tildes when referring to the shadow price system.

Note that for $N=1$, the system (13) reduces to the familiar state-costate system of optimal control, which allows one to interpret the variable $s$ as time, since the second equation of (13), combined with (1), implies that $\mathrm{d} \tilde{x} / \mathrm{d} s=F=\mathrm{d} \tilde{x} / \mathrm{d} t$. This interpretation cannot be made in the situation with two or more players.

In our analysis of solutions to the shadow price system (13), three manifolds are of central importance. The first is the shadow price dynamic nullcline

$$
D^{*}=\left\{(x, p): N q^{*}(x, p)-g(x)=0\right\},
$$

which gives the location of all possible steady states.

The other two are the horizontal and vertical nullclines $A_{1}^{*}, A_{2}^{*}$ of the shadow price system, given as

$$
A_{k}^{*}=\left\{(x, p): h_{k}^{*}(x, p)=0\right\}, \quad k=1,2 .
$$

Given our definition of strategies as piecewise differentiable, equilibrium strategies must be differentiable and continuous almost everywhere, and thus for almost all $x$ satisfy equation (12). We thus focus on the analysis of system (13) and its solution trajectories, segments of which we will be patching together to construct globally defined strategies. Such patching cannot be arbitrary, and we need to derive conditions which ensure that the strategies thus obtained are MPE.

Proposition 3.2 (First jump condition). Let $V(x)$ be the value function of a $M P E$ equilibrium, $\tilde{x} \in \mathcal{X}$ and $U$ a neighbourhood of $\tilde{x}$. If $V$ is continuous in $U$ and differentiable in $U \backslash\{\tilde{x}\}$ but not at $\tilde{x}$, then

$$
\lim _{x \uparrow \tilde{x}} G\left(x, V^{\prime}(x)\right)=\lim _{x \downarrow \tilde{x}} G\left(x, V^{\prime}(x)\right) .
$$



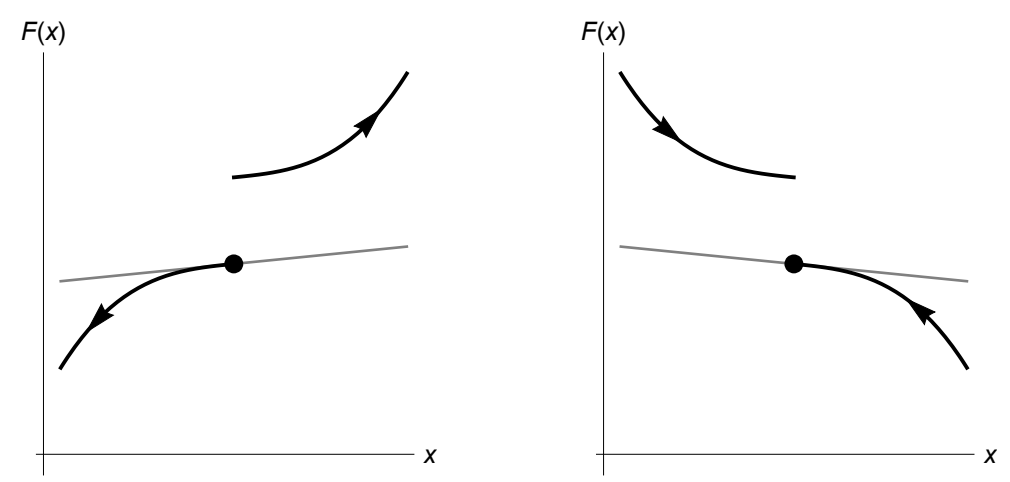

Figure 2: Possible steady state discontinuity configurations of $F(x)$ at a feedback Nash equilibrium.

The first jump condition follows immediately from Proposition 3.1 and continuity of value.

We first argue that stabilisation by discontinuous strategies is only possible if the stabilised point is reached asymptotically from one side.

Proposition 3.3 (Second jump condition). Suppose that $\phi$ is a strategy profile associated with an MPE, such that the following hold. There is $x \in \mathcal{X}$ and a neighbourhood $U$ of $x$ such that $F(y, \phi(y))$ is continuous at all points $y \in U$ except $x$, such that $F^{-}(x) \geq 0 \geq F^{+}(x)$, with at least one of the inequalities strict.

Then either $F^{-}(x)=0$ or $F^{+}(x)=0$.

Thus, a discontinuity in which the dynamics push (weakly) inwards from both sides must have one of the branches of the dynamics end exactly on the shadow price dynamic nullcline. The right panel of Figure 2 illustrates. The left panel demonstrates the implication of Proposition 2.7.

The logic is straightforward. Stabilisation at $x$ by discontinuous strategies which reach $x$ in finite time leads to a 'chattering' solution at $x$, and the payoffs reflect that. Thus, at $x$, the flow payoff to each player is a convex combination of $\tilde{u}_{i}\left(x, \phi_{i}^{-}(x)\right)$ and $\tilde{u}_{i}\left(x, \phi_{i}^{+}(x)\right)$, where $\phi_{i}^{+}(x)<\phi_{i}^{-}(x)$. Because of concavity of flow utility, any player could then do better by strategies which involve playing a convex combination of $\phi_{i}^{+}(x)$ and $\phi_{i}^{-}(x)$ in a small neighbourhood of $x$, yielding a strictly higher payoff in the long run.

\section{Pollution game}

To derive global necessary conditions, we need to specify a more concrete setting. We thus focus on the canonical problem of international stock pollution abatement. ${ }^{18}$ The framework is close to that of Dockner and Long (1993), but

\footnotetext{
${ }^{18}$ However, note that similar results would follow in closely-related model of e.g. joint investment in a public good or renewable resource management.
} 
we depart from their linear-quadratic specification; note that our specification is clearly not tractable by traditional algebraic methods.

Setting. We take the state space and the action set as $\mathcal{X}=(0, X)$ and $\mathcal{Q}=[m, M]$ respectively. The assumption that these spaces are bounded is for technical reasons. ${ }^{19}$ The felicity of a player is additively separable in own action $q$ and the state, and iso-elastic in $q$ :

$$
u(x, q)=\frac{q^{1-\sigma}}{1-\sigma}-C(x)
$$

where $\sigma>1$. The interpretation is that a player receives a positive payoff flow from $q$, the consumption of fossil fuels, with inelastic fossil fuel demand. The player also suffers damages due to the accumulated stock of greenhouse gases $x$. The term $g(x)$ in the dynamic equation (1) reflects the rate of natural decay of carbon from the atmosphere.

Introduce the set of interior shadow prices $\mathcal{P}_{I}=\left(m^{*}, M^{*}\right)$, where $m^{*} \equiv-m^{-\sigma}$ and $M^{*} \equiv-M^{-\sigma}$, as well as the sets of lower and upper corner shadow prices as, respectively, $\mathcal{P}_{m}=\left(-\infty, m^{*}\right]$ and $\mathcal{P}_{M}=\left[M^{*}, \infty\right)$. If $p \in \mathcal{P}_{I}$, the optimal action, as specified in equation (10), is

$$
q^{*}(p)=(-p)^{-1 / \sigma} \in \operatorname{int} \mathcal{Q},
$$

where we have abused notation marginally as the optimal action does not depend on $x$. For the corner cases, $q^{*}(p)=m$ if $p \in \mathcal{P}_{m}$ and $q^{*}(p)=M$ if $p \in \mathcal{P}_{M}$. Introduce for $q \in \mathcal{Q}$ the inverse

$$
p^{*}(q)=-q^{-\sigma}
$$

of $q^{*}(p)$. Then $m^{*}=p^{*}(m)$ and $M^{*}=p^{*}(M){ }^{20}$

The game Hamilton function is of the form

$$
G(x, p)=\frac{q^{*}(p)^{1-\sigma}}{1-\sigma}-C(x)+p\left(N q^{*}(p)-g(x)\right) .
$$

In particular we have, for interior values of $p$, that

$$
G(x, p)=\frac{(-p)^{1-1 / \sigma}}{1-\sigma}-C(x)+p\left(N(-p)^{-1 / \sigma}-g(x)\right), \quad \text { if } p \in \mathcal{P}_{I} .
$$

\footnotetext{
${ }^{19}$ The typical assumption in settings such as ours is that both state and control space are the positive real half-line. The economics of the situation does not change if $X$ and $M$ are assumed sufficiently high, and $m$ sufficiently small.

${ }^{20}$ Recall that, except for $q^{*}(\cdot)$ and $p^{*}(\cdot)$, the asterisk denotes variables in the shadow price system framework: e.g. $m^{*}$ and $M^{*}$ are the shadow price counterparts of actions $m$ and $M$, respectively.
} 
The shadow price vector field is of the form

$$
\frac{\mathrm{d} x}{\mathrm{~d} s}=h_{1}^{*}(x, p)=\frac{\partial G}{\partial p}, \quad \frac{\mathrm{d} p}{\mathrm{~d} s}=h_{2}^{*}(x, p)=\rho p-\frac{\partial G}{\partial x},
$$

where for $p \in \mathcal{P}_{I}$

$$
h_{1}^{*}(x, p)=K(-p)^{-1 / \sigma}-g(x), \quad h_{2}^{*}(x, p)=\left(\rho+g^{\prime}(x)\right) p+C^{\prime}(x),
$$

with $K=N-(N-1) / \sigma$, and

$$
\begin{aligned}
& h_{1}^{*}(x, p)=N m-g(x) \quad \text { for } p \in \mathcal{P}_{m}, \quad \text { and } \\
& h_{1}^{*}(x, p)=N M-g(x) \quad \text { for } p \in \mathcal{P}_{M},
\end{aligned}
$$

while $h_{2}^{*}$ is as in equation (20) in both cases.

The shadow price vector field is discontinuous at the boundary of the interior region $\mathcal{X} \times \mathcal{P}_{I}$, and its divergence is $\rho$. This implies in particular that there cannot be limit cycles in $\mathcal{X} \times \mathcal{P}_{I}$ (Wagener, 2003). ${ }^{21}$

The following result will be useful:

Lemma 4.1. $G(x, p)$ is convex in $p$ for $p \in \mathcal{P}_{I}$. For given $x$, if there exists $\tilde{p} \in \mathcal{P}_{I}$ satisfying $h_{1}^{*}(x, \tilde{p})=0$, then $G(x, \tilde{p})$ is a minimum point of $\tilde{p} \mapsto G(x, \tilde{p})$.

Note that while this is immediate for the single-player control problem, it need not hold for the non-cooperative case.

In order to make our points as cleanly as possible, we make some simplifying assumptions. $^{22}$ We start by imposing three conditions on the uncontrolled dynamics $g(x)$ and the state cost flow $C(x)$.

Assumption 4.1. The decay $g(x)$ is a bounded and continuously differentiable function of $x$ that satisfies $g(0)=0$ and $g^{\prime}(x)>0$ for all $x>0$.

The boundedness assumption is not restrictive, as the state space is bounded, but technically important. ${ }^{23}$

Assumption 4.2. The state cost flow $C(x)$ is a bounded and twice continuously differentiable function that satisfies $C(x) \geq 0, C^{\prime}(x)>0$ and $C^{\prime \prime}(x) \geq 0$ for $x \geq 0$.

The first inequality states that the stock variable $x$ is a public bad, while the second and third state that the marginal costs of the stock level are always positive and (weakly) increasing.

\footnotetext{
${ }^{21}$ If fossil fuel demand is sufficiently elastic, so that $K<0$, the structure of the game changes qualitatively; in particular, there can be multiple equilibria in continuous strategies (Wirl, 2014). This case remains work in progress.

${ }^{22}$ Alternative assumptions would imply slightly different global necessary conditions and would modify our results. However, the style of the analysis would remain the same.

${ }^{23}$ The decay function $g(x)$ can be interpreted as a production function: natural decay reduces the amount of pollution, hence produces a public good.
} 
Assumption 4.3. The elasticity of marginal decay is always smaller than the elasticity of marginal costs:

$$
\frac{x g^{\prime \prime}(x)}{g^{\prime}(x)}<\frac{x C^{\prime \prime}(x)}{C^{\prime}(x)} \quad \text { for all } x>0
$$

This assumption will be seen to imply that there is only a single interior singularity of the shadow price equation.

Nullclines. For ease of interpretation, as interior actions are monotonic in $p$, we map the shadow price vector field to a vector field in state-action variables (see Rincón-Zapatero et al., 1998; Dockner and Wagener, 2014), the action vector field. ${ }^{24}$ The implied system dynamics, valid for interior actions $q \in \operatorname{int} \mathcal{Q}$ and corresponding to equation (13), are then

$$
\frac{\mathrm{d} x}{\mathrm{~d} s}(s)=h_{1}(x(s), q(s)), \quad \frac{\mathrm{d} q}{\mathrm{~d} s}(s)=h_{2}(x(s), q(s)),
$$

where

$$
h_{1}(x, q) \equiv K q-g(x), \quad h_{2}(x, q) \equiv-\frac{q}{\sigma}\left(\rho+g^{\prime}(x)\right)+\frac{q^{1+\sigma}}{\sigma} C^{\prime}(x) .
$$

We can then also write the game Hamilton function in terms of the equilibrium action. For interior actions $q \in \operatorname{int} \mathcal{Q}$ it reads as

$$
\tilde{G}(x, q)=\frac{q^{1-\sigma}}{1-\sigma}-C(x)-q^{-\sigma}(N q-g(x))
$$

We can formulate the first jump condition, Proposition 3.2, in terms of $\tilde{G}$.

Lemma 4.2 (First jump condition). Let $\phi$ be an equilibrium strategy, $\tilde{x} \in X$ and $U$ a neighbourhood of $\tilde{x}$ in $\mathcal{X}$ such that $\phi(x) \in \operatorname{int} \mathcal{Q}$ for all $x \in U$. If $\phi$ is continuous in $U \backslash\{\tilde{x}\}$ but not at $\tilde{x}$, then

$$
\lim _{x \uparrow \tilde{x}} \tilde{G}(x, \phi(x))=\lim _{x \downarrow \tilde{x}} \tilde{G}(x, \phi(x)) .
$$

The vertical nullcline $A_{1}$ and the horizontal nullcline $A_{2}$ of the action vector field describe loci along which, respectively, $\mathrm{d} x / \mathrm{d} s=0$ and $\mathrm{d} q / \mathrm{d} s=0$. They are defined in terms of functions $a_{1}(x)$ and $a_{2}(x)$ as

$$
A_{i}=\left\{\left(x, a_{i}(x)\right): a_{i}(x) \in \operatorname{int} \mathcal{Q}\right\} .
$$

\footnotetext{
${ }^{24}$ We cannot dispose of the shadow price system due to the need to consider corner actions, i.e. because of the boundedness of the action space. This in turn is required to tackle transversality conditions without undue technical complications.
} 
The functions are given as

$$
a_{1}(x)=g(x) / K \quad \text { and } \quad a_{2}(x)=\left(\frac{\rho+g^{\prime}(x)}{C^{\prime}(x)}\right)^{1 / \sigma} .
$$

Lemma 4.3. The functions $a_{1}$ and $a_{2}$ have the following properties:

a. $a_{1}(0)=0$ and $a_{1}^{\prime}(x) \geq 0$ for all $x>0$;

b. $a_{2}(x)>0$ and $a_{2}^{\prime}(x)<0$ for all $x>0$.

The lemma is straightforward to prove. Note that the last statement, $a_{2}^{\prime}(x)<0$, is a consequence of Assumption 4.3. The implication is that the graphs of the functions $a_{1}$ and $a_{2}$ cross exactly once, at a point $(\bar{x}, \bar{q}) \cdot{ }^{25}$

The following lemma shows that, given the state cost flow and the natural dynamics, we can select the bounds on the control space and state space so that the shadow price vector field satisfies desirable properties:

Lemma 4.4. Given Assumptions 4.1-4.3, it is possible to choose the right hand point $X$ of the state space and the endpoints $m$ and $M$ of the action space such that the following relations are satisfied:

$$
\begin{gathered}
M<g(X) / N \\
M>a_{2}(0)=\left(\frac{\rho+g^{\prime}(0)}{C^{\prime}(0)}\right)^{1 / \sigma} ; \\
0<m<\left(\frac{\rho}{C^{\prime}(X)}\right)^{1 / \sigma}<a_{2}(X) .
\end{gathered}
$$

Proof. Choose first $M$ such that (24) is satisfied. As $g$ is increasing, it is then possible to choose $X$ such that (23) holds true. As $\rho>0$, it is then possible to choose $m$ such that (25) holds as well.

If $X, m$ and $M$ are chosen according to the lemma, this has a number of implications for the global behaviour of the shadow price vector field. Firstly, it implies that the vector field is outward pointing on the upper and lower boundary $p=m^{*}$ and $p=M^{*}$ of the interior region. Secondly, it is inward pointing at the left and right boundaries $x=0$ and $x=X$ : this justifies that no boundary values are specified there. Thirdly, it implies that the vector field has an interior steady state, which is unique by Lemma 4.3.

To formulate the final implication of the Lemma, we first introduce the stationary actions $d(x)=g(x) / N$ : i.e. if all players choose the action $q=d(x)$, then the state $x$ is stationary. As $N>K$, it is clear that $d(x)<a_{1}(x)$ for all $x>0$. We also introduce the corresponding stationary shadow price $d^{*}(x)=p^{*}(d(x))$. Let

\footnotetext{
${ }^{25}$ If the assumption is relaxed, the shadow price system may have multiple stationary points, and the analysis needs to be modified accordingly.
} 
$x_{m}$ and $x_{M}$ be respectively the steady states if all players play their minimal or their maximal action, that is, $m=d\left(x_{m}\right)$ and $M=d\left(x_{M}\right)$. We introduce the dynamic nullcline

$$
D=\left\{(x, d(x)): x_{m}<x<x_{M}\right\},
$$

and we note that the corresponding points $\left(x, d^{*}(x)\right)$ are on the shadow price dynamic nullcline $D^{*}$, introduced in (14).

Lemma 4.4 then implies that the slope $h_{2} / h_{1}$ of an orbit of the state-action system through the point $\left(x_{m}, m\right)$ is steeper than the slope $d^{\prime}\left(x_{m}\right)$ of the dynamic nullcline at that point.

We illustrate the dynamic nullcline and the two nullclines of the state-action system in Figure 3.

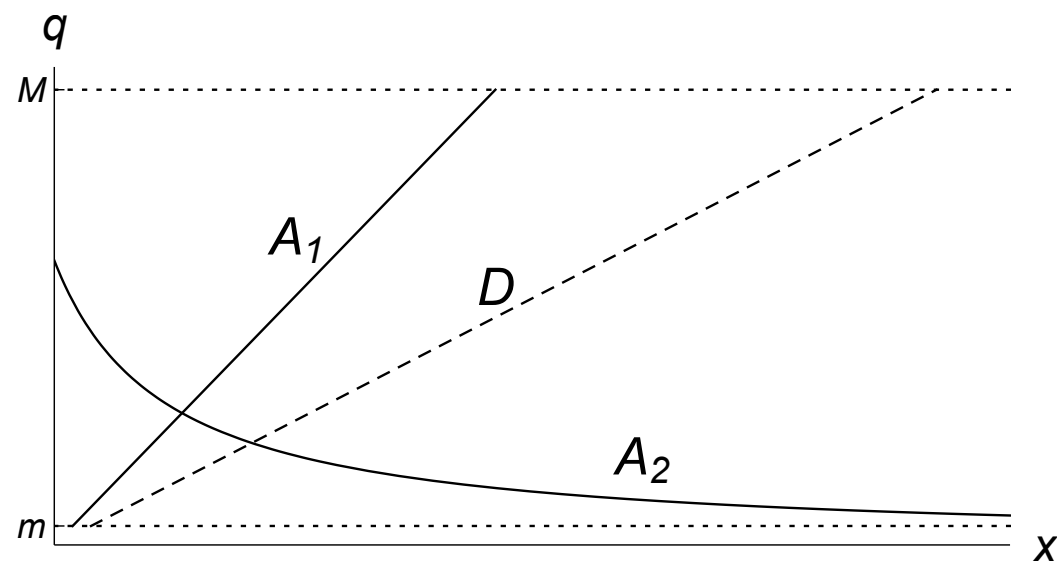

Figure 3: Nullclines $A_{1}$ and $A_{2}$ (solid) and dynamic nullcline $D$ (dashed).

\section{The phase diagram.}

Lemma 4.5. The interior steady state $(\bar{x}, \bar{q})$ of the shadow price system is a saddle point.

We denote the stable and unstable manifolds of the saddle $(\bar{x}, \bar{q})$ in the action system by $W^{s}$ and $W^{u}$ respectively. The following lemma allows us to construct the phase diagram.

Lemma 4.6. The stable and unstable manifolds $W^{u}$ and $W^{s}$ of the saddle $(\bar{x}, \bar{p})$ of the shadow price system can be represented as graphs $p=\Phi^{u *}(x)$ and $p=$ $\Phi^{s *}(x)$ of continuously differentiable functions that are defined on open intervals $\mathcal{X}^{u}, \mathcal{X}^{s} \subset \mathcal{X}$, both containing $\bar{x}$.

Introduce for $i \in\{s, u\}$ the functions $\Phi^{i}(x)=q^{*}\left(\Phi^{i *}(x)\right)$ for all $x \in \mathcal{X}^{i}$. Then we have the inequalities

$$
\Phi^{u}(x)<a_{1}(x)<\Phi^{s}(x) \quad \text { if } \quad x \in(0, \bar{x}) \cap \mathcal{X}^{u} \cap X^{s}
$$




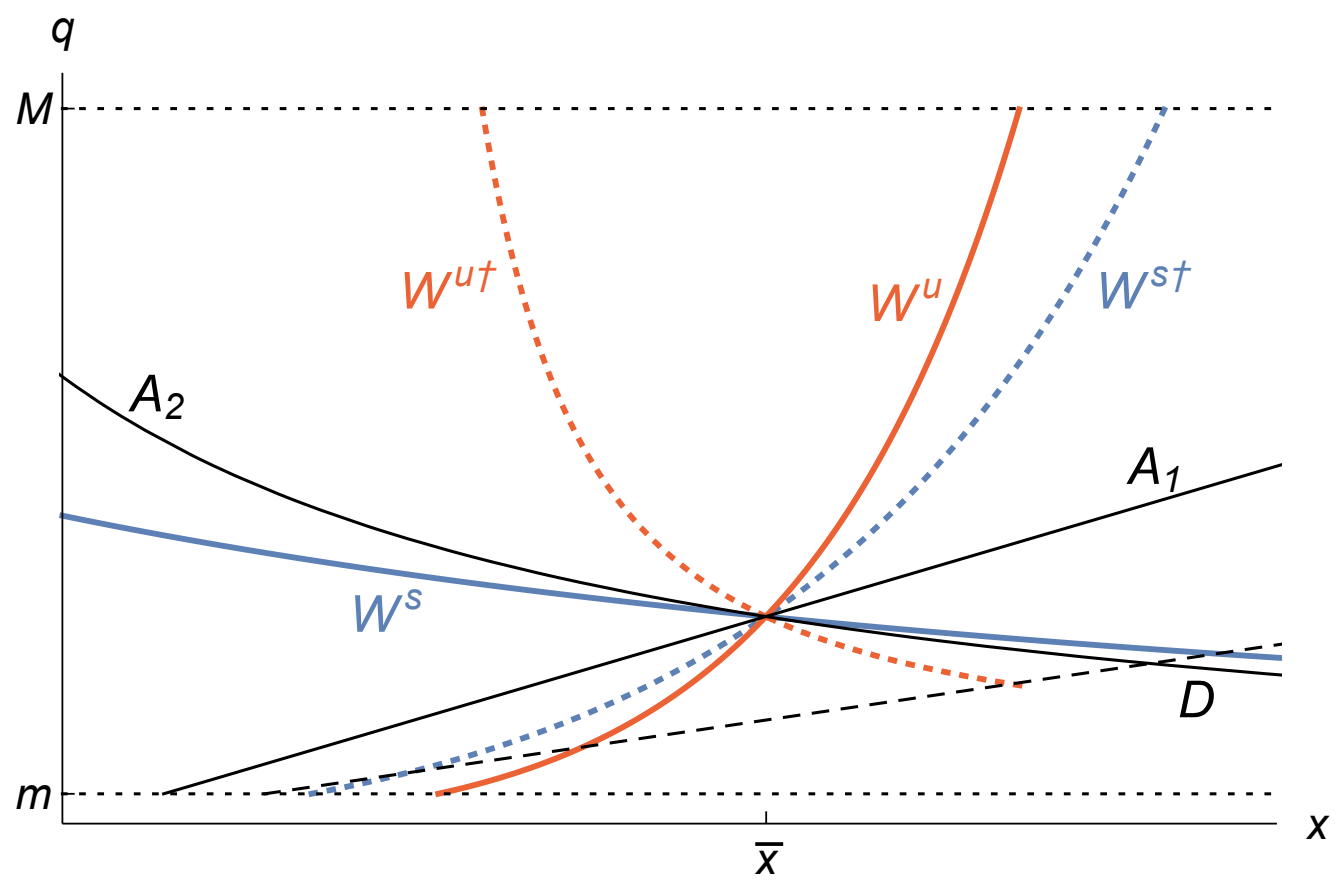

Figure 4: Phase diagram with key loci.

and

$$
\Phi^{s}(x)<a_{1}(x)<\Phi^{u}(x) \quad \text { if } \quad x \in(\bar{x}, X) \cap \mathcal{X}^{u} \cap X^{s} .
$$

Figure 4 plots the horizontal and vertical isoclines of the action system, the stable and unstable manifolds, and the dynamic nullcline. The strategies (described, piecewise, by trajectories of the action system) move the system to the right above the dynamic isocline, and to the left below. We assume the upper bound on the action variable $M$ is high enough to be ignored in the figure.

We next use transversality conditions to rule out some trajectories as resulting from MPE. Let moreover a one-sided neighbourhood of a point $\xi \in \mathcal{X}$ be a set of the form $(\xi-\delta, \xi]$ or $[\xi, \xi+\delta)$, for some $\delta>0$.

Lemma 4.7. Let $\phi$ be an admissible strategy that takes the value $m$ in a onesided neighbourhood of $x_{m}$ or the value $M$ in a one-sided neighbourhood of $x_{M}$. Then $\phi$ cannot be an equilibrium strategy.

The lemma does not rule out that $x_{m}$ appears as a — semi-stable — steady state of a Nash equilibrium, as it is possible that the equilibrium strategy is discontinuous at $x_{m}$ and such that $\phi(x) \rightarrow m$ as $x \downarrow x_{m}$. It does however imply that $x_{M}$ cannot occur as the steady state of a Nash equilibrium, as the corresponding discontinuity at $x_{M}$ is ruled out by the second jump condition. The asymmetry is a consequence of the fact that the dynamic nullcline $D$ is everywhere below the vertical isocline $A_{1}$. 
The continuous equilibrium. The next lemma confirms that the continuous equilibrium, which most of the literature has focused on, is unique.

Lemma 4.8. There is a unique continuous equilibrium, for which $\phi(x)=\Phi^{s}(x)$ if $x \in \mathcal{X}^{s}$.

Multiple continuous equilibria could be generated by sufficiently elastic demand (so that $K<0$; see Wirl, 2007), or by the unstable manifold $\Phi^{s}$ approaching $x=0$ above the dynamic nullcline (Rowat, 2007). In our setting, neither of these cases occurs, so that the continuous equilibrium is unique. ${ }^{26}$

Global necessary conditions. We now construct the set of equilibria with a continuous value function. First we consider the possibility that the equilibrium takes a corner value on an interval.

Proposition 4.9. Let $\phi$ be an equilibrium strategy, and let $\left(x_{0}, x_{1}\right) \subset \mathcal{X}$ be an interval such that $\phi(x)=M$ for $x \in\left(x_{0}, x_{1}\right)$ and $\phi(x)<M$ for $x<x_{0}$ and close to $x_{0}$ as well as for $x>x_{1}$ and close to $x_{1}$. Let

$$
p_{0}=\lim _{x \uparrow x_{0}} p^{*}(\phi(x)) \quad \text { and } \quad p_{1}=\lim _{x \downarrow x_{1}} p^{*}(\phi(x)) .
$$

Then the solution $V(x)$ of the differential equation

$$
\rho V=\frac{M^{1-\sigma}}{1-\sigma}-C(x)+V^{\prime}(x)(N M-g(x)), \quad V\left(x_{0}\right)=G\left(x_{0}, p_{0}\right) / \rho
$$

satisfies

$$
V\left(x_{1}\right)=G\left(x_{1}, p_{1}\right) / \rho .
$$

An analogous statement holds with $M$ replaced by $m$ and $\phi(x)<M$ replaced by $\phi(x)>m$.

This is a straightforward consistency condition that ensures continuity of the value function. It imposes a restriction on how strategies can feature actions taking corner values. ${ }^{27}$

We proceed to give a number of conditions that have to be necessarily satisfied by an equilibrium strategy $\phi$. These conditions are formulated geometrically in terms of 'forbidden regions' in the state-action space $\mathcal{X} \times \mathcal{Q}$ : the graph of an equilibrium strategy cannot have points in common with these regions.

The first of these forbidden regions, which will be denoted $R$, is given by all initial points $\left(x_{0}, p_{0}\right) \in \mathcal{X} \times \mathbb{R}$ such that the entire orbit $(x(s), p(s))$ of the shadow price system with this initial point satisfies the inequality

$$
G(x(s), p(s))>G\left(x(s), d^{*}(x)\right)
$$

\footnotetext{
${ }^{26}$ In the linear-quadratic framework with a unique continuous equilibrium, the strategy would of course be linear in the interior region.

${ }^{27}$ Such corner actions do not feature in the illustrated case we present in what follows, so the condition is never active in these. They could happen were the upper bound on actions $M$ low.
} 
whenever $s \geq 0$ and $x_{m} \leq x(s) \leq x_{M}$. Note that Proposition 3.1 and Lemma 4.1 imply that, for any given $x(s)$, the value is convex in $p(s)$, so that this inequality may be satisfied for low and/or high values of $p(s)$, but not necessarily for intermediate values.

The idea is the following. The second jump condition implies that if there were an equilibrium strategy $\phi$ corresponding to such an orbit, it would have to be continuous. As these orbits live entirely in the region with low or high values of $p$, we shall show that they eventually enter one of the corner regions, forcing $\phi(x)$ to take a corner value in a one-sided neighbourhood of either $x_{m}$ or $x_{M}$. But this is ruled out by the transversality condition, Lemma 4.7. Hence strategies featuring eventually very high or very low pollution rates are ruled out by this global necessary condition.

To characterise $R$, note that the orbits parametrising the graph of the continuous equilibrium $\Phi^{s}$ do not satisfy condition $(26)$, as $G(\bar{x}, \bar{p})<G\left(\bar{x}, d^{*}(\bar{x})\right.$ ) (Lemma 4.1 shows that for $\bar{x}, G(\bar{x}, p)$ has a minimum in $\mathcal{P}_{I}$ at $\left.p=\bar{p}\right)$. Together with the convexity of value, this implies that the region $R$ consists of two components, $R^{+}$and $R^{-}$, the first above the graph of $\Phi^{s}$, the other below it.

To describe the boundary of $R^{-}$, consider the intersection point $\left(a, d^{*}(a)\right)$ of the dynamic nullcline and the graph of $p^{*}\left(\Phi^{s}(x)\right)$, and the vertical line $x=a$ through this point. On this line, a point $(a, p)$ with $p$ large and negative is in $R^{-}$, whereas the intersection point $\left(a, d^{*}(a)\right)$ is not. Let $p_{b}$ be the supremum of the values of $p$ such that $(a, p) \in R^{-}$: then $p_{b}<d^{*}(a)$, and the boundary of $R^{-}$ equals the orbit through $\left(a, p_{b}\right)$, at least locally around this point.

This orbit has necessarily a point $\left(\hat{x}, d^{*}(\hat{x})\right)$ in common with $D^{*}$, otherwise $p_{b}$ would not be a supremum, but as the orbit has to remain below $D^{*}$, by definition of $R$, it has to be a point of tangency.

Definition 4.10. The lower limit strategy $\Phi^{\ell-}(x)$ is given by the orbit of the action system which extends out from $(\hat{x}, d(\hat{x}))$ when the orbit is in the interior region, and $\Phi^{\ell-}(x)=m$ elsewhere.

The lower limit strategy is uniquely defined, even if the orbit has multiple tangencies with $D .{ }^{28}$ Also, in general the lower limit strategy will not be an equilibrium strategy (because it will break the transversality condition for low $x$ ).

To describe the boundary of the upper component $R^{+}$in an analogous manner, we have to introduce the conjugate to the dynamic nullcline.

Definition 4.11. The conjugate shadow price dynamic nullcline $D^{* \dagger}$ is the set of points $\left(x, d^{* \dagger}(x)\right)$ satisfying $x_{m}<x<x_{M}, d^{* \dagger}(x) \neq d^{*}(x)$ and

$$
G\left(x, d^{* \dagger}(x)\right)=G\left(x, d^{*}(x)\right) .
$$

The conjugate dynamic nullcline $D^{\dagger}$ is the graph of $d^{\dagger}(x)=q^{*}\left(d^{* \dagger}(x)\right)$.

\footnotetext{
${ }^{28}$ In the illustrations, we plot the case with a single tangency, as in the linear-quadratic case (Dockner and Long, 1993).
} 
Then a boundary point of $R^{+}$can be found by taking the infimum of values of $p$ such that $(b, p) \in R^{+}$, where $b$ is the state coordinate of an intersection of the graph of $\Phi^{s}(x)$ with $D^{\dagger}$. The orbit extending from this point must have a point of tangency $\left(\check{x}, d^{\dagger}(\check{x})\right)$ with $D^{\dagger}$.

Definition 4.12. The upper limit strategy $\Phi^{\ell+}(x)$ is given by the orbit of the action system which extends out from $\left(\check{x}, d^{\dagger}(\check{x})\right)$ when the orbit is in the interior region, and $\Phi^{\ell+}(x)=M$ elsewhere.

We also define conjugates to the stable and unstable curves, which are such that the value of the Hamiltonian along a conjugate locus equals that along its counterpart:

Definition 4.13. The conjugate stable curve and conjugate unstable curve, $W^{s^{\dagger}}$ and $W^{u \dagger}$ respectively, are given by the sets of interior points $(x, q)$ satisfying either $(x, q)=(\bar{x}, \bar{q})$ or $x \neq \bar{x}$ and, respectively, $\tilde{G}(x, q)=\tilde{G}\left(x, \Phi^{s}(x)\right)$, $q \neq \Phi^{s}(x)$ and $\tilde{G}(x, q)=\tilde{G}\left(x, \Phi^{u}(x)\right), q \neq \Phi^{u}(x)$.

It follows immediately from the convexity of $G(x, p)$ in $p$ that, for any given $x$, at most one point can belong to $D^{\dagger}, W^{s \dagger}$, or $W^{u \dagger}$, and if the conjugate point exists, it must lie on the opposite side of $A_{1}$ relative to $D, W^{s}$ and $W^{u}$, respectively. Moreover, by the implicit function theorem the conjugate stable and unstable curves are differentiable curves in the interior. We plot these loci in Figure 4. Importantly, the conjugate stable and unstable curves $W^{s^{\dagger}}$ and $W^{u^{\dagger}}$ bracket the stable curve $W^{s}$ between them. To see this, introduce $G^{i}(x)=G\left(x, \Phi^{i *}(x)\right)$ and $p^{i}(x)=\left(G^{i}\right)^{\prime}(x) / \rho$ for $i \in\{s, s \dagger, u, u \dagger\}$. As the graphs of $\Phi^{s *}(x)$ and $\Phi^{u *}(x)$ are orbits, it follows that $p^{i}(x)=\Phi^{i *}(x)$ for $i \in\{s, u\}$. Moreover, for $x>\bar{x}$ we have the shadow price relation

$$
\frac{\mathrm{d} G^{u}}{\mathrm{~d} x}=p^{u}(x)>p^{s}(x)=\frac{\mathrm{d} G^{s}}{\mathrm{~d} x} .
$$

Since $G^{u}(\bar{x})=G^{s}(\bar{x})$, this implies that $G^{u \dagger}(x)=G^{u}(x)>G^{s}(x)=G^{s^{\dagger}}(x)$ for $x>\bar{x}$. By the convexity of $G(x, p)$ as a function of $p$, this consequently implies for $x>\bar{x}$ that

$$
\Phi^{u \dagger}(x)<\Phi^{s}(x)<a_{1}(x)<\Phi^{s \dagger}(x)<\Phi^{u}(x) .
$$

These inequalities are reversed for $x<\bar{x}$.

We now have the required tools to construct the set of equilibria when the value function is continuous (but strategies may be discontinuous). The following two propositions give global necessary conditions, showing particular regions in $(x, q)$-space through which no equilibrium strategy can pass.

Proposition 4.14. Suppose $\phi$ is a symmetric MPE. Then the graph of $\phi$ has no point in common with the set $R$. 


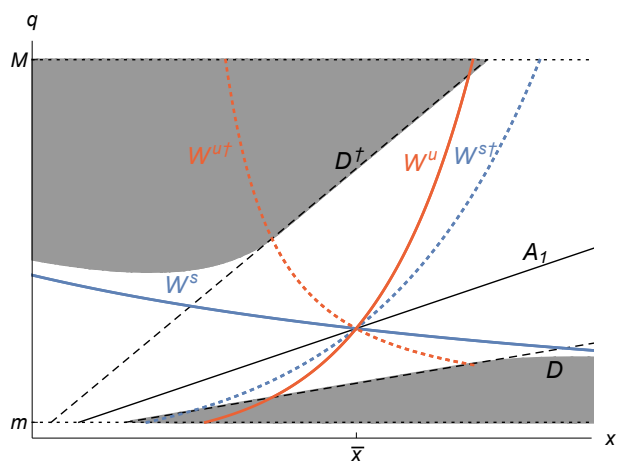

(a) Lemma 4.14

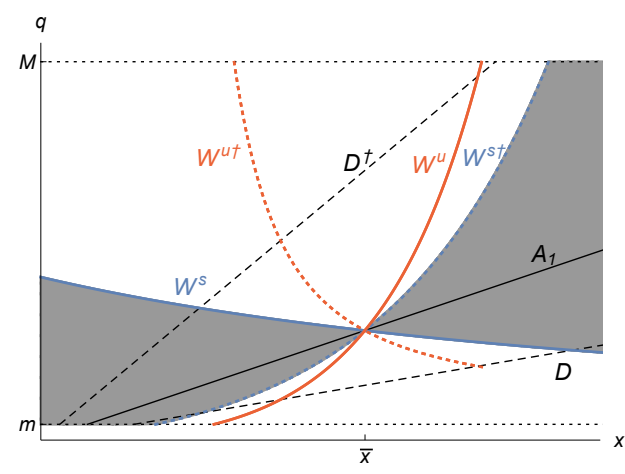

(b) Lemma 4.15

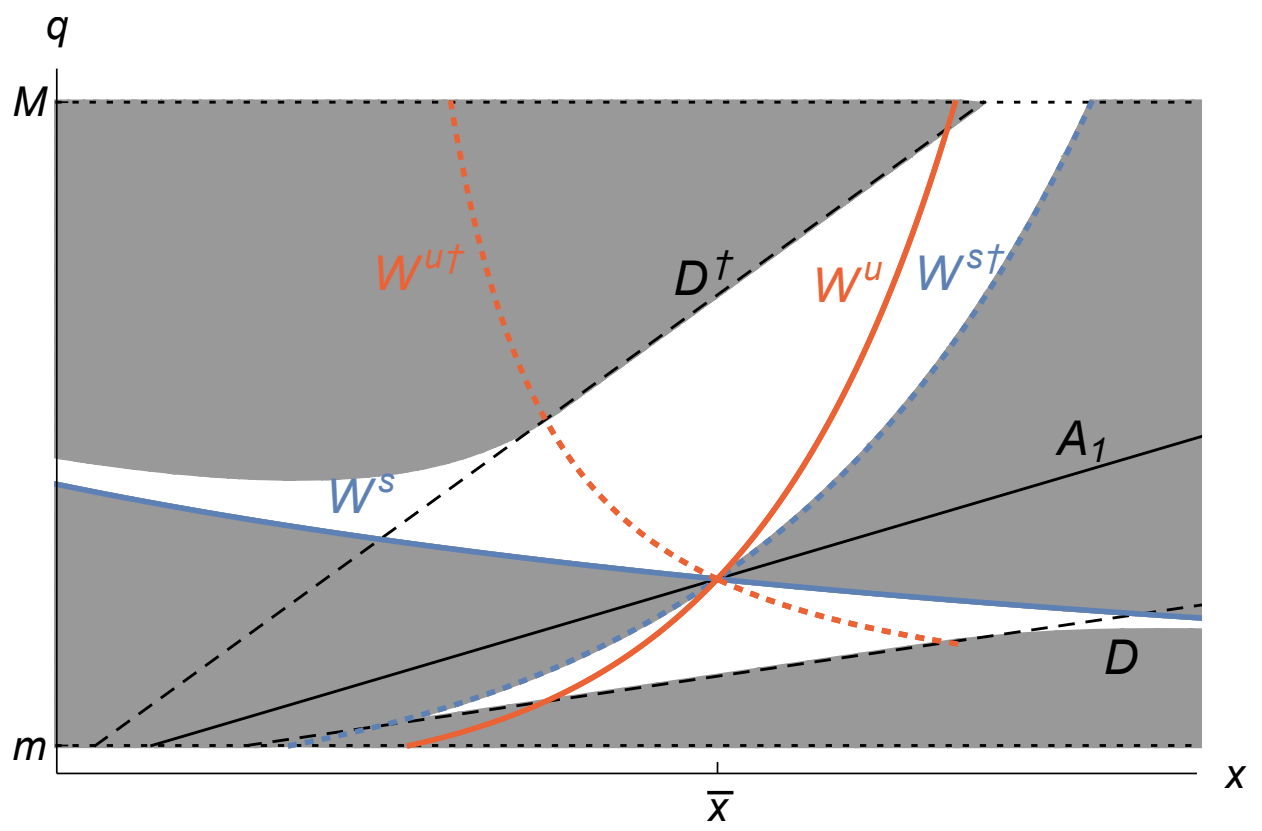

(c) All excluded regions

Figure 5: Regions excluded from MPNE strategies. 
The proposition is illustrated in Figure 5a.

We next rule out further orbits of the action system as forming parts of equilibria, by showing they cannot be extended to globally defined strategies. In particular, many sections of 'backward-bending' orbits can be ruled out:

Proposition 4.15. Let $\phi$ be an MPE associated with a value function that is continuous everywhere. Then $\phi(x) \notin \operatorname{int} \operatorname{co}\left(\Phi^{s}(x), \Phi^{s \dagger}(x)\right)$.

The backward-bending arcs which are excluded yield very low value, and cannot be extended across the entire state space given that value functions must be continuous. Figure 5b shows the regions excluded by Lemma 4.15, and Figure $5 \mathrm{c}$ shows all excluded regions.

Results. We can now state our first key result: a necessary and sufficient condition for Markov-perfect Nash equilibria.

Theorem 4.16. Let $\phi$ be a piecewise continuously and boundedly differentiable MPE strategy. Then $\phi$ satisfies the following necessary conditions:

a. the Hamilton-Jacobi-Bellman condition, equation (11), which is valid whenever $\phi$ is differentiable and interior;

b. the jump conditions of Propositions 3.2 and 3.3, which are valid at points of non-differentiability of $\phi$;

c. the global necessary conditions of Propositions 4.9, 4.14 and 4.15.

Conversely, if $\phi$ satisfies these conditions, it is a symmetric Markov perfect Nash equilibrium.

To sketch out the proof: we apply results showing that the optimal solution to player $i$ 's problem is given by the unique viscosity solution $V$ to a HamiltonJacobi-Bellman equation. We demonstrate that a strategy satisfying all the conditions will give rise to a value function $V_{i}$, which is a viscosity solution of said equation, and must thus coincide with $V$; this establishes sufficiency. Conversely, by uniqueness of $V$, the conditions are also necessary.

Our second main result is a characterisation of all MPE with no discontinuity in value. Before stating our result formally, we state its contents by describing such trajectories geometrically.

In Figure 6, compared to Figure 5c, two additional regions are shown. As before, the interior of the dark grey regions cannot contain equilibrium strategies. We shall describe the possible shapes of equilibrium strategies going from small to large values of $x$.

For small values of $x$, they are constrained to the closure of the medium grey region, bounded below by $W^{s}$ and $D^{\dagger}$ and above by the trajectory that is tangent to $D^{\dagger}$. Restricted to this region, the strategy must be continuous. 


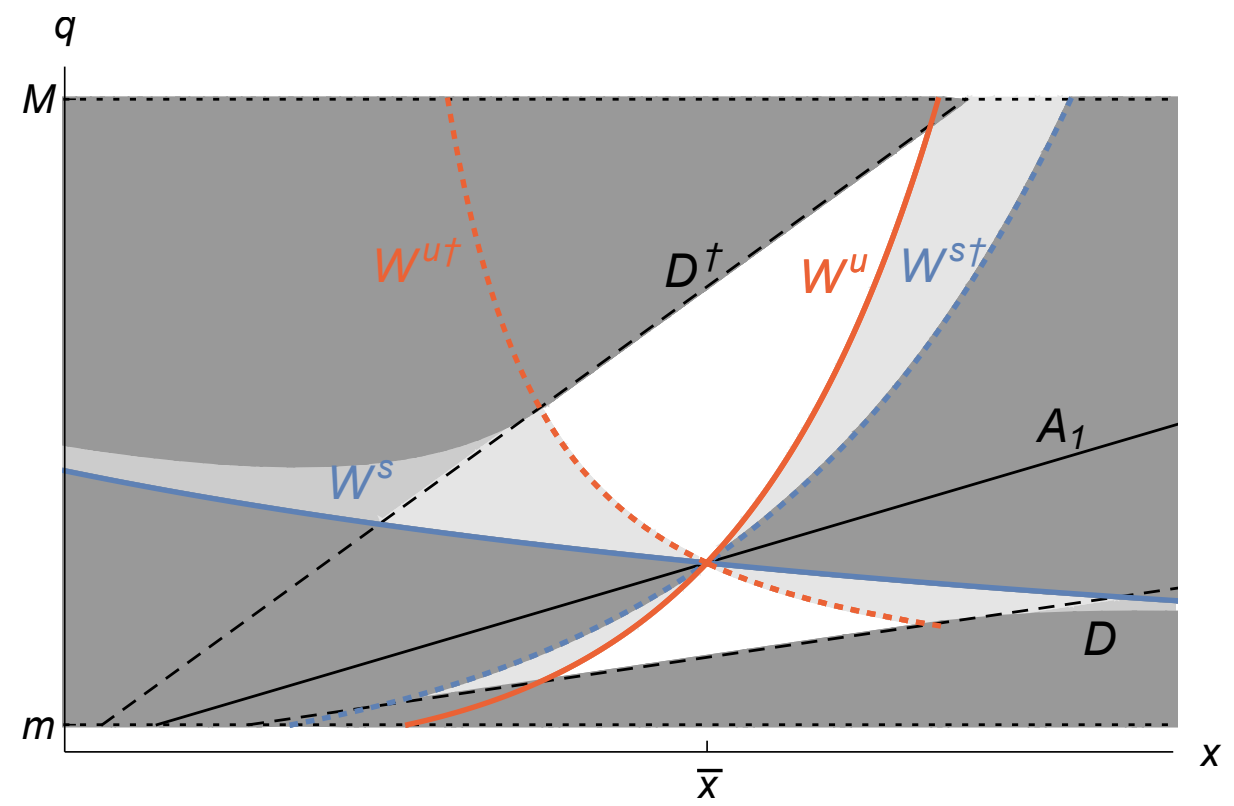

Figure 6: Global restrictions on MPNE strategies.

The strategy then enters the light grey region; if it is not located on $W^{s}$ it may have an even or an odd number of jumps in the part of the region for which $x$ is lower than the steady state value $\bar{x}$. If it has an even number of jumps, it may end on $W^{s}$, and then it is constrained to pass through the steady state $(\bar{x}, \bar{q})$, or it ends above $W^{s}$ and it has to pass into the white region. If it has an odd number of jumps, it has to end on $W^{u}$ and is again constrained to pass through the steady state $(\bar{x}, \bar{q})$.

If the strategy enters the white region that is above $W^{s}$, bounded below by $W^{u \dagger}$ and $W^{u}$, it must have an odd number of jumps, eventually ending in the white region that is below $W^{s}$, bounded above by $W^{u}$ and $W^{u \dagger}$. From here on the discussion proceeds symmetrically to the above: it passes into the light grey region that allows still for an even number of jumps, and then into the medium grey region where no jumps are allowed any more.

If the strategy passes by $W^{u}$ or $W^{s}$ to the steady state, then it can continue by going along the same manifold, or it may switch to the other. If it switches, it is continuous but not differentiable at $\bar{x}$ (see Dockner and Wagener, 2014). Again, from here on the discussion is symmetric to what has been before.

Our claim is that Figure 6 and the accompanying verbal description provides necessary and sufficient conditions for Markov perfect Nash equilibrium strategies with continuous values.

The preceding analysis allows us to define the accessible region (to repeat, all but the dark grey regions in Figure 6) for equilibrium strategies. 
Definition 4.17. The accessible region $\Xi$ is defined as

$$
\begin{aligned}
\Xi \equiv & \left\{(x, q): \min \left\{\Phi^{s}(x), \Phi^{s \dagger}(x)\right\} \leq q \leq \max \left\{d^{\dagger}(x), \Phi^{p \dagger}(x)\right\}\right\} \\
& \cup\left\{(x, q): \min \left\{\Phi^{s}(x), \Phi^{s \dagger}(x)\right\} \geq q \geq \max \left\{d(x), \Phi^{p}(x)\right\}\right\} .
\end{aligned}
$$

A point $(x, q) \in \Xi$ is called accessible.

We now state our second key result.

Theorem 4.18. For any accessible point $(x, q)$, there exists a symmetric MPE strategy $\phi$ such that $\phi(x)=q$.

\section{$5 \quad$ Welfare-ranking equilibria}

We can rank equilibria in terms of welfare. Clearly, as we only consider symmetric equilibria, a strict ranking implies Pareto-dominance.

We first give a lemma which is helpful in constructing equilibria in discontinuous strategies and in Pareto-ranking different equilibria. This follows immediately from Proposition 3.1 and Lemma 4.1.

Lemma 5.1. For any $x$, take two $M P E \phi^{1}, \phi^{2}$ such that either $\phi^{1}(x)>\phi^{2}(x)>$ $a_{1}(x)$ or $\phi^{1}(x)<\phi^{2}(x)<a_{1}(x)$. Then the implied value functions satisfy $V^{1}(x)>V^{2}(x)$.

Pareto-ranking the equilibria is easy with the help of Lemma 5.1 and Figure 6: for any initial state $x_{0}$, strategies specifying an action further out from $A_{1}$ dominate strategies specifying an action closer to $A_{1}$.

Thus, for any initial state $x_{0}$, a Pareto-dominant equilibrium $\phi$ prescribes the action furthest out from the nullcline $A_{1}$. Which equilibria are Pareto-dominant depends on $x_{0}$ : this is $\Phi^{\ell+}$ if $x_{0} \leq \min \{\hat{x}, \check{x}\}$ and $\Phi^{\ell-}$ if $x_{0} \geq \max \{\hat{x}, \check{x}\}$. If $\check{x}>\hat{x}$, then either $\Phi^{\ell+}$ or $\Phi^{\ell-}$ is Pareto-dominant if $x_{0} \in(\hat{x}, \check{x}) .{ }^{29}$ Finally, if $\check{x}<\hat{x}$, then for $x_{0} \in(\check{x}, \hat{x})$ the Pareto-dominant strategy has a discontinuity at $x_{0}$, with $\lim _{x \rightarrow x^{-}} \phi(x)=d^{\dagger}\left(x_{0}\right), \lim _{x \rightarrow x^{+}} \phi(x)=d\left(x_{0}\right)$. Fixing the prescribed action at $x_{0}$, the strategies can then be extended for all $x$ using the procedure described in the previous section.

For $x_{0} \in[\check{x}, \hat{x}]$, if non-empty, the Pareto-dominant strategy induces $x_{0}$ to be a steady state. For $x_{0} \in[\check{x}, \hat{x})$, this steady state is semi-stable: a trajectory of the state dynamics converging to $x_{0}$ from the left reaches the state in finite time. A trajectory starting at a point $x_{0}+\varepsilon$ drifts very slowly up, for arbitrarily small $\varepsilon>0$. That is, from a Pareto-optimal steady state, a deviation downward would trigger a discontinuous increase in other players' emission rates; a deviation upward would trigger no reduction in emission rates. For $x_{0}=\hat{x}$, the steady

\footnotetext{
${ }^{29}$ Close to $x=\hat{x}$, a strategy with $\phi(x)=\Phi^{\ell+}(x)$ yields higher value; close to $x=\check{x}$, a strategy with $\phi(x)=\Phi^{\ell-}(x)$ does.
} 
state is stable, but the approach from $x>x_{0}$ occurs at a rate below exponential; that is, very slowly. See Figure 7.

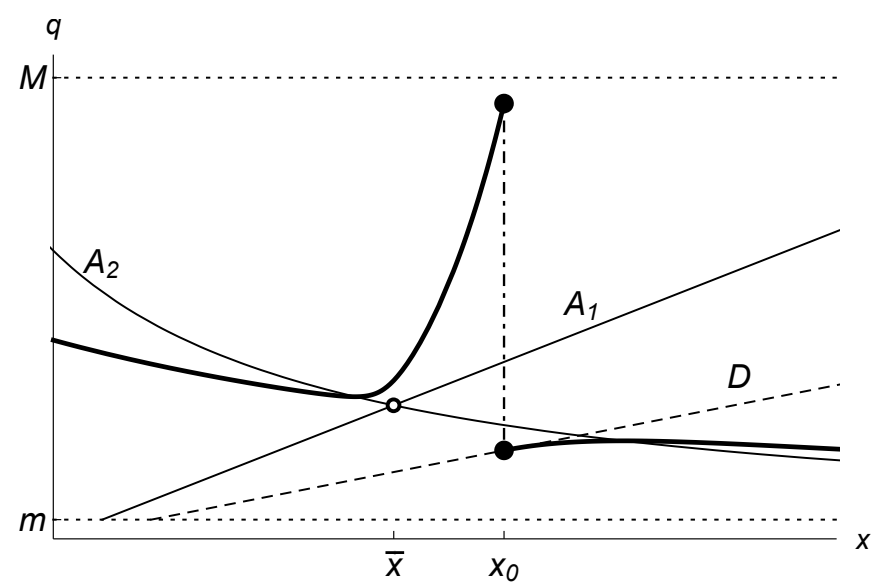

Figure 7: Pareto-dominant equilibrium for $x_{0}=\hat{x}$.

It is these asymmetries in equilibrium responses to deviations which sustain Pareto-optimal steady states. If $[\check{x}, \hat{x}]$ is empty, then no steady state is Paretooptimal; however, any Pareto-optimal strategy will lead to a steady state which is supported by a similar discontinuity.

The last observation draws attention to an interesting property of the welfareranking: no equilibrium is Pareto-dominant for all $x$. This raises the issue of players preferring to reoptimise on the path following a deviation. Under rational expectations, any renegotiation would be foreseen. The definition and study of 'renegotiation-proof' MPE is beyond the scope of the current paper, however.

We finally obtain our third key result.

Theorem 5.2. Suppose $\phi$ is a continuous-value MPE strategy. Then, $\phi$ weakly Pareto-dominates the unique continuous strategy $\Phi^{s}$ for any $x$.

In other words, out of the large set of continuous-value equilibria (but with discontinuities in strategies allowed), any discontinuous equilibrium is at least as good as the continuous one.

Note that the continuous equilibrium is the focus of most of the extant literature on differential games of public goods investment. In this specific model, it yields the worst welfare outcome of all possible symmetric MPE. Indeed, for any $x$, there are many equilibria which strictly dominate $\Phi^{s}$ in terms of welfare. This raises an issue with the positive importance of the continuous equilibrium: we should not expect to observe it being played in any context in which the players understand the game and are able to communicate before the start of play. The continuous equilibrium is also defective from a normative point of view, of course.

The intuition for our result is simple. At $\bar{x}$, the worst possible equilibrium value is given by a strategy passing through $(\bar{x}, \bar{q})$ - such as the continuous strategy. 
This anchors the continuous strategy as giving the worst value for all $x$ : no strategy which yields a value lower than $\Phi^{s}$ for some $x \neq \bar{x}$ can be extended across $\bar{x}$. This results from the equilibrium value being continuous: if a strategy yielding lower value than $\Phi^{s}$ for some $x_{0} \neq \bar{x}$ could be extended to $\bar{x}$, it would have to have equal value with $\Phi^{s}$ for some $x$ in between. This is not possible in our context: strategies cannot cross, and the saddlepoint nature of the shadow price system guarantees that values cannot cross via discontinuities either. Thus, it is the requirement of subgame perfection, i.e. that the equilibrium must be well-defined for any possible history, which excludes the possibility of strategies worse than $\Phi^{s}$.

\section{Discussion}

We have demonstrated an extension of the theory of differential games in a simple, univariate context which allows for the full treatment of discontinuous strategies, revealing a large set of novel equilibria. These equilibria have been almost entirely unnoticed in the previous literature. Moreover, we have demonstrated a new technique of constructing equilibria without having to resort to particular functional specifications. Our methods also make it very easy to numerically compute equilibria.

Our results have implications on empirical work studying public goods games. ${ }^{30}$ In contexts, whether experimental or observational, which fit our assumptions, and in which the agents can be expected to be sufficiently sophisticated and able to communicate with one another, there would be little reason to expect to observe the Pareto-dominated continuous equilibrium being played. An estimation which does not take the possibility of discontinuous equilibria into account is thus potentially misspecified. It would also be of interest to look for evidence to confirm (or not) our findings in experimental settings or in real-world contexts.

We have used our methods to derive results in a differential game of climate change mitigation, with a relatively general functional specification. We expect the gist of our results to carry over to other games involving dynamic investment into public goods, such as games of noncooperative renewable resource management, or of joint investment into common projects. A particular case of interest would involve problems with nonconvex dynamics.

The techniques we demonstrate are quite general and can be used to construct equilibria for other classes of differential games. They are also easy to apply, and can thus be employed in the numerous applications in the literature - in problems involving advertising, dynamic oligopolies, resource extraction, patent races, and many other applications - which have to date focused on continuous equilibria. Our findings call for a reassessment of results obtained in these various applications.

\footnotetext{
${ }^{30}$ Examples of such work include the recent contributions by Vespa (2020) and Battaglini et al. (2016)
} 
Our construction makes welfare-ranking the various equilibria, given the state of the economy, obvious. However, given any equilibrium which is selected (say, the Pareto-optimal MPE for the initial state), the economy will then evolve along the equilibrium path. This evolution may take the economy to a state in which the selected equilibrium is dominated by some other equilibrium. This raises the problem of renegotiation-proofness (Bernheim and Ray, 1989; Farrell and Maskin, 1989), but in a dynamic context, with an endogenously evolving state variable. Thus, dynamic equilibrium selection and renegotiation proofness present interesting avenues for future research.

We have fully characterised the set of symmetric MPE. Of course, there may well exist many more asymmetric equilibria. Whether our methods can be extended to construct these also is an interesting question. Characterising the entire set of MPE, symmetric or not, would shed further light on the structure of public goods games and would complete the strand of reseach on multiple equilibria in such games started by Dockner and Long (1993). ${ }^{31}$

Similarly, this paper has focused on the univariate case, as it is particularly easy to study. An obvious question is whether our methods can be extended also to multivariate settings. We leave this question for future research.

In terms of climate change, our results place particular emphasis on the role of climate negotiations as an equilibrium selection mechanism. In our simple setting, the undominated equilibria (given an initial state) involve setting a stabilisation target for long-run atmospheric carbon concentrations, reached by a relatively high-emission path followed by rapid decarbonisation. Interestingly, recent debates in climate policy have focused on long-run carbon concentration levels or on temperature targets. However, more research is required to ascertain whether this intuition also holds if our simplifying assumptions are relaxed. Given the urgency of the climate change problem, we feel this should be a research priority.

\section{A Proofs}

\section{A.1 Proof of Proposition 2.3}

Proof. Consequence of Filippov selection; see step 2 in proof of Theorem 2.1 of Barles et al. (2013).

\section{A.2 Proof of Proposition 2.7}

Proof. Let $\left(q^{-}, q^{+}, \mu\right) \in \mathcal{Q} \times \mathcal{Q} \times[0,1]$ be such that

$$
0=\mu f_{i}^{-}\left(\tilde{x}, q^{-}\right)+(1-\mu) f_{i}^{+}\left(\tilde{x}, q^{+}\right) .
$$

\footnotetext{
${ }^{31}$ De Frutos and Martín-Herrán (2018) is one of the few papers focusing on asymmetric equilibria.
} 
Then

$$
0=\mu q^{-}+(1-\mu) q^{+}-g(\tilde{x}) .
$$

Introducing $\bar{q}=\mu q^{-}+(1-\mu) q^{+}$shows that $x(t)$ is also a possible trajectory for $(\bar{q}, \bar{q}, \mu)$ for $t \in\left[0, t_{1}\right]$. Because of strict concavity of $\tilde{u}_{i}$ in $q_{i}$, we have

$$
\mu \tilde{u}_{i}\left(\tilde{x}, q^{-}\right)+(1-\mu) \tilde{u}_{i}\left(\tilde{x}, q^{+}\right) \leq \tilde{u}_{i}(\tilde{x}, \bar{q}) .
$$

Hence $(\bar{q}, \bar{q}, \mu)$ dominates $\left(q^{-}, q^{+}, \mu\right)$.

Moreover, as $\tilde{u}_{i}(\tilde{x}, q)$ is increasing in $q$, there is a largest value $q^{m}$ of $q$ such that $f_{i}^{-}(\tilde{x}, q) \leq 0$ and $f_{i}^{+}(\tilde{x}, q) \geq 0$. Setting $\mu=1$ if $f_{i}^{-}\left(\tilde{x}, q^{m}\right)=0$ and $\mu=0$ otherwise, we have that $\tilde{u}_{i}\left(\tilde{x}, q^{m}\right) \geq \tilde{u}(\tilde{x}, \bar{q})$. This proves the result.

\section{A.3 Proof of Proposition 3.3}

Proof. The argument is entirely analogous to the proof of Proposition 2.7: the direction of the dynamics does not imply material changes.

\section{A.4 Proof of Lemma 4.1}

Proof. We have that

$$
G_{p p}(x, p)=\frac{1-N(1-\sigma)}{\sigma^{2}}(-p)^{-1 / \sigma-1} \quad \text { if } p \in \mathcal{P}_{I},
$$

and $G_{p p}(x, p)=0$ if $p \in \mathcal{P}_{m} \cup \mathcal{P}_{M}$. Given our assumption $\sigma>1$, it follows that $G_{p p} \geq 0$ everywhere, and $G$ is convex as a function of $p$ in $\mathcal{P}_{I}$.

It is easy to confirm that for fixed values of $x, \tilde{p}$ defined by $K q^{*}(\tilde{p})-g(x)=0$ is a critical point $G_{p}(x, \tilde{p})=0$ of $G(x, p)$ if $\tilde{p} \in \mathcal{P}_{I}$. For values of $x$ such that $\tilde{p} \in \mathcal{P}_{I}$, this critical point is in the interior region where $G_{p p}>0$. Hence the critical point is isolated and the global minimiser of $G(x, p)$.

\section{A.5 Proof of Lemma 4.5}

Proof. For $i=1,2$ we can write $h_{i}(x, q)=\psi_{i}(x, q)\left(q-a_{i}(x)\right)$ with $\psi_{i}(x, q)>0$ differentiable. At the steady state $(\bar{x}, \bar{q})$, where $\bar{q}=a_{1}(\bar{x})=a_{2}(\bar{x})$ and $\bar{\psi}_{i}=$ $\psi_{i}(\bar{x}, \bar{q})$, we have

$$
D h(\bar{x}, \bar{q})=\left(\begin{array}{cc}
-\bar{\psi}_{1} a_{1}^{\prime} & \bar{\psi}_{1} \\
-\bar{\psi}_{2} a_{2}^{\prime} & \bar{\psi}_{2}
\end{array}\right)
$$

and

$$
\operatorname{det} D h(\bar{x}, \bar{q})=\bar{\psi}_{1} \bar{\psi}_{2}\left(a_{2}^{\prime}(\bar{x})-a_{1}^{\prime}(\bar{x})\right) .
$$

Lemma 4.3 states that $a_{2}^{\prime}(x) \leq 0<a_{1}^{\prime}(x)$ for all $x$. It follows that the determinant is negative and that the steady state is a saddle. 


\section{A.6 Proof of Lemma 4.6}

Proof. Since $\bar{\psi}_{1} \neq 0$, the vector $(0,1)$ is not an eigenvector of $D h(\bar{x}, \bar{q})$. By the invariant manifold theorem (Hirsch et al., 1977), locally around $(\bar{x}, \bar{q})$ the stable and unstable manifolds can be represented as graphs of continuously differentiable functions of $x$.

Introduce the set $B=B^{-} \cup B^{+}$, where

$$
\begin{aligned}
& B^{-}=\left\{(x, q): x<\bar{x}, a_{1}(x)<q<M\right\}, \\
& B^{+}=\left\{(x, q): x>\bar{x}, m<q<a_{1}(x)\right\},
\end{aligned}
$$

and $F=F^{-} \cup F^{+}$, where

$$
\begin{aligned}
& F^{-}=\left\{(x, q): x<\bar{x}, m<q<a_{1}(x)\right\} \\
& F^{+}=\left\{(x, q): x>\bar{x}, a_{1}(x)<q<M\right\} .
\end{aligned}
$$

See Figure 8.

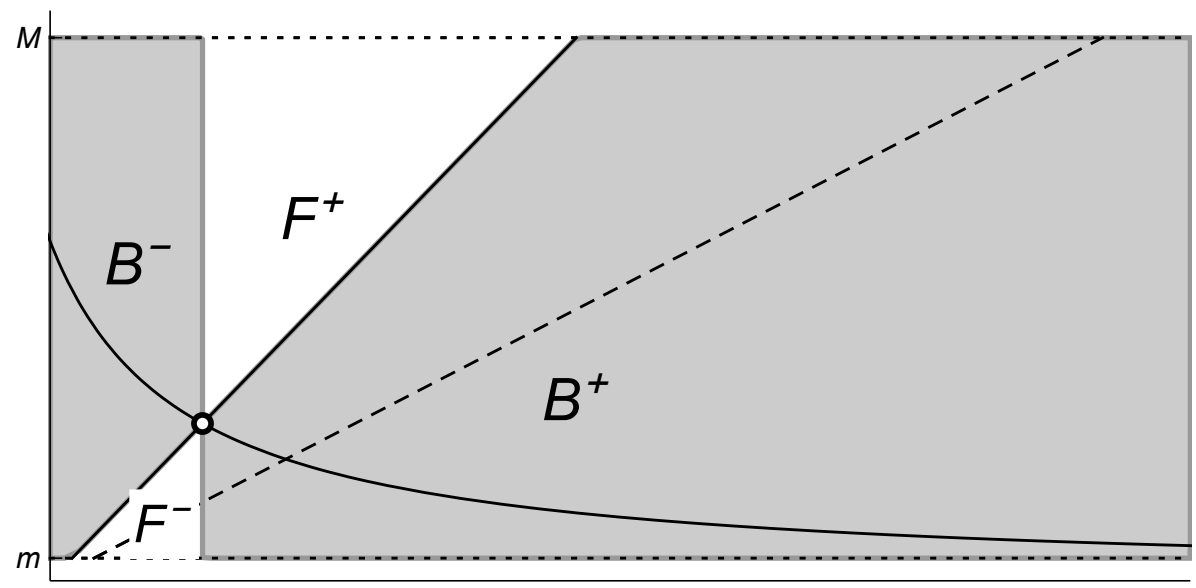

Figure 8: Forward trapping regions $F^{-}$and $F^{+}$and backward trapping regions $B^{-}$and $B^{+}$.

The vector field $h$ is outward pointing at the boundary of $B$ and inward pointing at the boundary of $F$. Hence $F$ is forward invariant and $B$ backward invariant, that is, all future points of a trajectory of $h$ with initial point in $F$ are also located in $F$, and all past points of a trajectory with initial point in $B$ are also located in $B$.

This implies that excepting $(\bar{x}, \bar{q})$, all points of the stable manifold $W^{s}$ are contained in $B$ and all points of the unstable manifold $W^{u}$ are in $F$. This implies the inequalities. As $h_{1} \neq 0$ in $F \cup B$, it follows that the local invariant manifolds can be extended over all of $\mathcal{X}$. 


\section{A.7 Proof of Lemma 4.7}

Proof. Let $U=\left(x_{M}-\delta, x_{M}\right]$ be a one-sided neighbourhood of $x_{M}$ such that $\phi(x)=M$ for all $x \in U$. As $g^{\prime}\left(x_{M}\right)>0$, the steady state $x_{M}$ is attracting: if $x(0) \in U$, then $x(s) \rightarrow x_{M}$ as $s \rightarrow \infty$. We want to show that the trajectory $x(s)$ cannot occur if $\phi$ is a Markov perfect symmetric equilibrium strategy.

Arguing by contradiction, let $V_{i}(x)$ denote the value function for player $i$. Restricted to $U$, the Hamilton-Jacobi equation for player $i$ takes the form

$$
\rho V_{i}=\frac{M^{1-\sigma}}{1-\sigma}-C(x)+V_{i}^{\prime}(x)(N M-g(x)) .
$$

Repeating the arguments that led to the derivation of the shadow price system, we remark that the graph $\left(x, V_{i}(x)\right)$ coincides with solution trajectories $(x(t), v(t))$ of the system

$$
\dot{x}=N M-g(x), \quad \dot{v}=\rho v-\frac{M^{1-\sigma}}{1-\sigma}+C(x) .
$$

This system has a saddle point $\left(x_{M}, v_{M}\right)$, where $v_{M}=\rho^{-1}\left(M^{1-\sigma} /(1-\sigma)-\right.$ $\left.C\left(x_{M}\right)\right)$ is the steady state value at $x_{M}$. The unstable manifold of this steady state is the vertical line $x=x_{M}$. Hence a solution trajectory of the system that has a finite limit as $x \rightarrow x_{M}$ is necessarily located on the stable manifold of the saddle.

The invariant manifold theorem implies that the stable manifold is smooth, that is, as often differentiable as $g$ and $C$, and that it is tangent to the stable eigenspace. From this, we infer that the value function, which is necessarily finite, is smooth, and that its graph equals the stable manifold of the saddle. Its gradient is computed from the stable eigenspace. The matrix $A$ of the linearisation of the system at the saddle takes the form

$$
A=\left(\begin{array}{cc}
-g^{\prime}\left(x_{M}\right) & 0 \\
C^{\prime}\left(x_{M}\right) & \rho
\end{array}\right) .
$$

The stable eigenvalue is $-g^{\prime}\left(x_{M}\right)$, the associated stable eigenspace the span of the vector $\left(\rho+g^{\prime}\left(x_{M}\right),-C\left(x_{M}\right)\right)$, and hence

$$
V_{i}^{\prime}\left(x_{M}\right)=-\frac{C^{\prime}\left(x_{M}\right)}{\rho+g^{\prime}\left(x_{M}\right)}=-a_{2}\left(x_{M}\right)^{-\sigma}<M^{*} .
$$

This implies that $\phi\left(x_{M}\right)<M$, which is a contradiction.

The proof in the situation that $\phi(x)=m$ in a one-sided neighbourhood of $x_{m}$ is entirely analogous. 


\section{A.8 Proof of Lemma 4.8}

Proof. The graph of a continuous equilibrium strategy is invariant under the shadow price vector field. If the strategy is not identical with $\Phi^{s}$ in the interior region $\mathcal{X} \times \operatorname{int} \mathcal{Q}$, there is a point $\left(x_{0}, q_{0}\right)$ on the graph such that the forward trajectory through that point does not converge to $(\bar{x}, \bar{q})$. Let $p_{0}$ denote the associated value of the shadow price.

We continue to use the notations $B^{ \pm}, F^{ \pm}$, introduced in the proof of lemma 4.6. Consider first the situation that $\left(x_{0}, q_{0}\right) \in B^{-} \cup F^{+}$, that is, the region bounded by the axis $x=0$, the lower boundary $q=m$, the upper boundary $q=M$ and the vertical nullcline $A_{1}$.

By the Poincaré-Bendixson theorem, the forward limit set of the trajectory is a steady state, a limit cycle, or the trajectory escapes from every compact subset. We have already noted that the shadow price system cannot have limit cycles. By assumption, the trajectory cannot converge to the interior steady state. Hence it leaves the interior at a point $(x(\tilde{s}), q(\tilde{s}))$.

When discussing leaving trajectories, the shadow price representation has to be considered. Assume the orbit leaves the interior region through the upper boundary and passes into the upper corner region $\mathcal{X} \times \mathcal{P}_{M}$ : that is, assume $p(\tilde{s})=M^{*}$ and $\mathrm{d} p / \mathrm{d} s(\tilde{s})>0$. As $\mathrm{d} p / \mathrm{d} s(s)>0$ if $p(s) \in \mathcal{P}_{M}$, the trajectory remains in the region $\mathcal{X} \times \mathcal{P}_{M}$. Hence the associated strategy $\phi$ satisfies $\phi(x)=M$ for $x(\tilde{s})<x<x_{M}$. But this is ruled out for an equilibrium strategy by Lemma 4.7. Hence no trajectory starting in $B^{-} \cup F^{+}$not on the stable manifold of $(\bar{x}, \bar{q})$ can correspond to a continuous equilibrium strategy.

The situation that $\left(x_{0}, q_{0}\right) \in B^{+} \cup F^{-}$is entirely analogous.

Finally, a trajectory in the interior region but not in $B^{-} \cup B^{+} \cup F^{-} \cup F^{+}$for $t=0$, that is, which starts on one of the boundaries, is in the latter region for some $t>0$, and the previous argument applies.

\section{A.9 Proof of Proposition 4.14}

Proof. Assume that $\phi$ is an equilibrium strategy whose graph partially lies in $R$. We show first that $\phi$ has to be continuous inside $R$. For assume that $\phi$ is discontinuous at a point $x_{1}$ such that $\left(x_{1}, \phi^{+}\left(x_{1}\right)\right) \in R$. As $\phi^{+}\left(x_{1}\right)<d\left(x_{1}\right)$, by definition of the conjugate dynamic isocline, $\phi^{-}\left(x_{1}\right)>d^{\dagger}\left(x_{1}\right)$ or $\phi^{-}\left(x_{1}\right)=$ M. In particular, $F^{-}=\lim _{x \uparrow x_{1}} f(x, \phi(x))>0$ and $F^{+}=\lim _{x \downarrow x_{1}} f(x, \phi(x))<$ 0 . But this is ruled out by Lemma 3.3. The argument for the situation that $\left(x_{1}, \phi^{-}\left(x_{1}\right)\right) \in R$ is analogous.

Take $\left(x_{0}, p_{0}\right) \in R^{-}$such that $p_{0} \in \mathcal{P}_{I}$, and consider the orbit $(x(s), p(s))$ of the shadow price system with this initial point. We claim that the orbit has to intersect the line $p=m^{*}$ at a point $\left(x\left(s_{m}\right), m^{*}\right)$ with $s_{m}>0$. To show this, consider the set

$$
T^{-}=\left\{(x, p) \in R^{-}: x \leq x_{0}, p \geq m^{*}\right\} .
$$


Since $h_{1}^{*}(x, p)<0$ for all $(x, p) \in R^{-}$, the shadow price vector field is pointing into $T$ along $x=x_{0}$. As $A_{1}$ has no points in common with $T$ (which must lie below $D$, thus below $A_{1}$ ) the shadow price vector field has no steady states in $T$. Neither has it invariant circles. Hence the orbit has to leave $T$ through the curve $p=m^{*}$ at some point $\left(x\left(s_{m}\right), m^{*}\right)$.

If $x\left(s_{m}\right)=x_{m}$, the slope of the orbit can be at most have the value $\left(d^{*}\right)^{\prime}\left(x_{m}\right)$ of the slope of $D^{*}$ at the point of exit, or, equivalently, the slope of the stateaction orbit is at most the value $d^{\prime}\left(x_{m}\right)$ of the slope of $D$ at $\left(x_{m}, m\right)$. Introduce therefore

$$
\Delta(x)=\frac{h_{2}(x, d(x))}{h_{1}(x, d(x))}-d^{\prime}(x) .
$$

As $h_{1}(x, d(x))<0$, the vector field $h$ is inward pointing if $\Delta(x)>0$. A straightforward computation shows that

$$
\Delta\left(x_{m}\right)=\frac{C^{\prime}\left(x_{m}\right)}{N-1}\left(\frac{\rho+d^{\prime}\left(x_{m}\right)}{C^{\prime}\left(x_{m}\right)}-m^{\sigma}\right) \geq \frac{d^{\prime}\left(x_{m}\right)}{N-1}>0,
$$

where the penultimate inequality is a direct consequence of Lemma 4.4. Hence the slope of the orbit is too large, and $x\left(s_{m}\right)>x_{m}$.

As the lower corner region $\mathcal{X} \times \mathcal{P}_{m}$ is forward invariant for the shadow vector field, we have $p(s)<m^{*}$ and $q(s) \equiv q^{*}(p(s))=m$ for all $s>s_{m}$. Hence for $s>s_{m}$, we have that $x(s)$ is a solution of

$$
x^{\prime}(s)=N m-g(x(s)),
$$

and therefore $x(s) \rightarrow x_{m}$ as $s \rightarrow \infty$. We conclude that $\phi(x)=m$ for all $x$ in the one-sided neighbourhood $\left[x_{m}, x\left(s_{m}\right)\right)$ of $x_{m}$. But this is ruled out by Lemma 4.7 .

The argument for an orbit with initial point $\left(x_{0}, p_{0}\right) \in R^{+}$is slightly different. The orbit is shown to intersect $p=M^{*}$ at some $s=s_{M}>0$ by considering $T^{+}\left\{(x, p) \in R^{+}: x \geq x_{0}, p \leq M^{*}\right\}$ and noting that the shadow price vector field is inward pointing along $x=x_{0}$. The point of intersection $\left(x\left(s_{M}\right), M^{*}\right)$ satisfies $x\left(s_{M}\right) \leq x_{M}^{\dagger}$, where $d^{\dagger}\left(x_{M}^{\dagger}\right)=M$. Since $d^{\dagger}(x)>a_{1}(x)>d(x)$ for all $x_{m} \leq x \leq x_{M}$, we have $d\left(x_{M}^{\dagger}\right)<M=d\left(x_{M}\right)$. As $d$ is increasing, $x_{M}^{\dagger}<x_{M}$.

The remainder of the argument is as before: $p(s)>M^{*}$ and $q(s) \equiv q^{*}(p(s))=M$ for all $s>s_{M}$, hence for these values of $s$ we have that $x(s)$ is a solution of $x^{\prime}(s)=N M-g(x(s))$ and $x(s) \rightarrow x_{M}$ as $s \rightarrow \infty$. Hence $\phi(x)=M$ on a one-sided neighbourhood of $x_{m}$, which is ruled out by Lemma 4.7.

\section{A.10 Proof of Proposition 4.15}

Proof. Note that if $\phi(x) \in \operatorname{co}\left(\Phi^{s}(x), \Phi^{s \dagger}(x)\right)$ and $V(x)=\tilde{G}(x, \phi(x)) / \rho$ is the value associated to $\phi$, then $V(x)<\tilde{G}\left(x, \Phi^{s}(x)\right)=\tilde{G}\left(x, \Phi^{s \dagger}(x)\right)$. Introduce also $V^{s}(x)=\tilde{G}\left(x, \Phi^{s}(x)\right)$. 
By contradiction: assume that $\phi$ is an MPE such that for some $0<x_{0}<\bar{x}$ we have $\phi\left(x_{0}\right) \in \operatorname{co}\left(\Phi^{s}\left(x_{0}\right), \Phi^{s \dagger}\left(x_{0}\right)\right)$, and therefore $V\left(x_{0}\right)<V^{s}\left(x_{0}\right)$. We claim that $V(x)<V^{s}(x)$ for all $x_{0} \leq x \leq \bar{x}$.

Assume that there exists a smallest value $x_{1}$ in the interval $\left[x_{0}, \bar{x}\right]$ for which the inequality $V(x)<V^{s}(x)$ fails. Then we have for $x_{0} \leq x<x_{1}$ necessarily that $\Phi^{s \dagger}(x)<\phi(x)<\Phi^{s}(x)$. Since $q^{*}$ and its inverse $p^{*}$ are increasing functions, this implies

$$
V\left(x_{1}\right)=V\left(x_{0}\right)+\int_{x_{0}}^{x_{1}} p^{*}(\phi(\xi)) \mathrm{d} \xi<V^{s}\left(x_{0}\right)+\int_{x_{0}}^{x_{1}} p^{*}\left(\Phi^{s}(\xi)\right) \mathrm{d} \xi=V^{s}\left(x_{1}\right) .
$$

Hence $V(x)<V^{s}(x)$ for all $x \in\left[x_{0}, \bar{x}\right]$, and in particular, $V(\bar{x})<V^{s}(\bar{x})$. But as $q=\Phi^{s}(\bar{x})$ minimises $\tilde{G}(\bar{x}, q)$, we should have that $V(\bar{x}) \geq V^{s}(\bar{x})$. As it is impossible that both inequalities hold, the strategy $\phi$ cannot exists.

The case that $\bar{x}<x_{0}$ is treated similarly, again by integrating towards $\bar{x}$.

\section{A.11 Proof of Theorem 4.16}

Proof. We want to apply Barles et al. (2014, Theorem 4.4), which states that, given a number of conditions, the value function of the optimisation problem of player $i$ is continuous and the unique viscosity solution of a certain HamiltonJacobi equation. ${ }^{32}$

To make this statement precise, we introduce the superdifferential $D^{+} V(x)$ of a continuous function $V$ at a point $x$ as

$$
D^{+} V(x)=\left\{p \in \mathbb{R}: \lim _{y \rightarrow x} \sup _{y \in \Omega} \frac{u(y)-u(x)-p(x-y)}{|x-y|} \leq 0\right\} .
$$

The subdifferential $D^{-} V(x)$ is defined similarly, with sup replaced by inf and $\leq$ by $\geq$ (see e.g. Bardi and Capuzzo-Dolcetta, 2008, Chapter II). We have that $p \in D^{+} V(x)$ if and only if there is a continuously differentiable function $\psi$ such that $\psi^{\prime}(x)=p$ and $V(x)-\psi(x)$ has a local maximum at $x$. An analogous characterisation exists for subdifferentials.

The function $V$ is a viscosity supersolution of the equation

$$
\rho V(x)-H\left(x, V^{\prime}(x)\right)=0
$$

at $x$, if

$$
\rho V(x)-H(x, p) \geq 0,
$$

for all $p \in D^{-} V(x)$, and a subsolution if

$$
\rho V(x)-H(x, p) \leq 0
$$

\footnotetext{
${ }^{32}$ Although Barles et al. (2014) introduce multiple value functions, as a consequence of Proposition 2.7 , all of these coincide in our context.
} 
for all $p \in D^{+} V(x)$. Finally, $V$ is a viscosity solution at $x$ if it is there both a viscosity supersolution and a viscosity subsolution.

The conditions of Theorem 4.4 of Barles et al. (2014), formulated for our context, are as follows. For every $0 \leq j \leq J$, we have:

H1 the state dynamics $f_{i, j}\left(x, q_{i}, \mu_{i}\right)$ is bounded and satisfies a Lipschitz condition in $x$, uniformly with respect to $\left(q_{i}, \mu_{i}\right)$;

$\mathrm{H} 2$ the flow payoff $u_{i, j}\left(x, q_{i}, \mu_{i}\right)$ is uniformly continuous and bounded;

H3 the felicity function $\tilde{u}_{i}\left(x, q_{i, j}\right)$ is concave in $q_{i, j}$;

H4 for $x \in \mathcal{I}_{j}$ there are $\delta>0$ and $q_{i, j}^{ \pm}, \tilde{q}_{i, j}^{ \pm} \in \mathcal{Q}$ such that

$$
\tilde{f}_{i, j-1}\left(x, q_{i}^{-}\right)=\tilde{f}_{i, j}\left(x, \tilde{q}_{i}^{-}\right)=-\delta
$$

and

$$
\tilde{f}_{i, j-1}\left(x, q_{i}^{+}\right)=\tilde{f}_{i, j}\left(x, \tilde{q}_{i}^{+}\right)=\delta .
$$

Conditions H1, H2 and H3 are satisfied in our context, H4 not necessarily. To be able to apply Theorem 4.4 of Barles et al. (2014), we therefore have to adapt the context.

Consider first the local controllability assumption H4, and assume that it fails to be satisfied at an interface point $x \in \mathcal{I}_{j}$ for all actions $\left(q_{i}, \mu_{i}\right)$. Introduce the spaces $\mathcal{Y}_{j}$ and $\mathcal{Z}_{j}$ such that

$$
\mathcal{X}=\mathcal{Y}_{j} \cup \mathcal{I}_{j} \cup \mathcal{Z}_{j}
$$

where $\mathcal{Y}_{j} \cup \mathcal{I}_{j}=\cup_{k=0}^{j-1} \mathcal{X}_{k}$ and $\mathcal{Z}_{j} \cup \mathcal{I}_{j}=\cup_{k=j}^{J} \mathcal{X}_{k}$. Failing of local controllability implies that for $x \in \mathcal{I}_{j}$ either (1) $\tilde{f}_{i, j-1} \leq 0$, or (2) $\tilde{f}_{i, j-1} \geq 0$, or (3) $\tilde{f}_{i, j} \geq 0$, or (4) $\tilde{f}_{i, j} \leq 0$. In all cases, the condition is to hold for all actions $\left(q_{i}, \mu_{i}\right)$.

Consider first the situation that (1) holds. Then the optimisation problem in $\mathcal{Y}_{j}$ is independent of whatever happens in $\mathcal{Z}_{j}$. Denote its value by $V_{i, j-1}(x)$.

If (3) holds as well, the optimisation problem in $\mathcal{Z}_{j}$ is independent of what happens in $\mathcal{Y}_{j}$, and the problem decomposes into two simpler problems.

If (3) does not hold, the optimisation problem in $\mathcal{Z}_{j}$ is then a problem with potential exit through $\mathcal{I}_{j}$, with boundary condition $V_{i, j}(x)=V_{i, j-1}(x)$ at the moment of exit. The value function $V_{i, j}$ is then the unique viscosity solution of the HJB equation on $\mathcal{Z}_{j}$ with the given boundary condition (see Fleming and Soner (2006), Theorems 13.1(a) and 14.1).

Next, consider the situation that (1) does not hold, but that (3) holds. This is analogous to the previous situation, only with the roles of $\mathcal{Y}_{j}$ and $\mathcal{Z}_{j}$ interchanged.

The last situation is that neither (1) nor (3) hold, but that either (2) or (4) holds. 
If (2) holds, the optimisation problem in $\mathcal{Z}_{j}$ is an exit problems with boundary value $b_{j}(x)=\left(M^{1-\sigma} /(1-\sigma)-C(x)\right) / \rho$, which gives rise to a value function $V_{i, j}$ in $\mathcal{Z}_{j}$, and which satisfies $V_{i, j}(x) \leq b_{j}(x)$. Consequently, the optimisation problem in $\mathcal{Y}_{j}$ is an exit problem with boundary condition $V_{i, j-1}(x)=V_{i, j}(x)$.

The situation that (4) holds is symmetrical.

We can therefore assume that the local controllability condition holds, if necessary by restricting to a smaller domain.

Introduce the local Hamilton functions

$$
H_{i, j}\left(x, p_{i}\right)=H_{i}\left(x, p_{i} ; \phi_{-i, j}\right)=\frac{\sigma}{1-\sigma}\left(-p_{i}\right)^{1-1 / \sigma}-C(x)+p_{i}\left(\Phi_{-i, j}(x)-g(x)\right) .
$$

As the 'singular dynamics' case is never optimal, Barles et al. (2014) implies that a continuous function $V$ is a viscosity solution of the Hamilton-Jacobi-Bellman equation

$$
\rho V(x)-H_{i}\left(x, V^{\prime}\right)=0
$$

on $\mathcal{X}$, if it is a viscosity solution of

$$
F_{i, j}\left(x, V, V^{\prime}\right)=\rho V-H_{i, j}\left(x, V^{\prime}\right)=0 \quad \text { if } x \in \operatorname{int} \mathcal{X}_{j}
$$

and, for $x \in \mathcal{I}_{j}$, a viscosity supersolution of

$$
F^{*}{ }_{i, j}\left(x, V, V^{\prime}\right)=\rho V-\min \left\{H_{i, j-1}\left(x, V^{\prime}\right), H_{i, j}\left(x, V^{\prime}\right)\right\}=0
$$

and a viscosity subsolution of

$$
F_{* i, j}\left(x, V, V^{\prime}\right)=\rho V-\max \left\{H_{i, j-1}\left(x, V^{\prime}\right), H_{i, j}\left(x, V^{\prime}\right)\right\}=0 .
$$

Moreover, the value function $V_{i}$ of player $i$ is also a viscosity solution of equation (29) (Barles et al., 2014). Since Theorem 4.4 of Barles et al. (2014) implies that the viscosity solution of that equation is unique in our context, it follows that $V_{i}$ is the value function of player $i$.

We have therefore to show that a strategy $\phi$ that satisfies all the necessary conditions gives rise to a viscosity solution of the Hamilton-Jacobi-Bellman equation.

First we construct the value function $W$ associated to $\phi$ as the solution to the differential equation

$$
\rho W(x)=\frac{\phi(x)^{1-\sigma}}{1-\sigma}-C(x)+W^{\prime}(x)(N \phi(x)-g(x)) .
$$

It follows from the necessary conditions that $W$ is well-defined, continuous, and piecewise differentiable. We shall show that it is a viscosity solution of (29), and therefore is equal to the value function $V_{i}$ of player $i$.

Note that for every $j$ and every $x \in \operatorname{int} \mathcal{X}_{j}$, we have by construction that $W$ is 
differentiable at $x, \phi_{i, j}(x):=\phi(x)=\left(-W^{\prime}(x)\right)^{-1 / \sigma}, \Phi_{-i, j}(x)=(N-1) \phi(x)$ and

$$
\rho W(x)-H_{i, j}\left(x, W^{\prime}(x) ; \phi_{-i, j}(x)\right)=0 .
$$

Hence restricted to int $\mathcal{X}_{j}$ the function $W$ is a viscosity solution of (30) by construction.

Consider now the situation at an arbitrary interface point $x \in \mathcal{I}_{j}$. Introduce the quantities $p^{-}$and $p^{+}$as

$$
\lim _{y \uparrow x} W^{\prime}(y)=p^{-}, \quad \lim _{y \downarrow x} W^{\prime}(y)=p^{+} .
$$

Note that by construction

$$
\rho W(x)-H_{i, j-1}\left(x, p^{-}\right)=\rho W(x)-H_{i, j}\left(x, p^{+}\right)=0 .
$$

The first situation to be investigated is $p^{-}<p^{+}$. Then $D^{+} W(x)=\emptyset$ and $D^{-} W(x)=\left[p^{-}, p^{+}\right]$. As $D^{+} W(x)$ is empty, $W$ is automatically a subsolution at $x$. We have to show that it is also a supersolution at $x$.

Let $q^{-}=q^{*}\left(p^{-}\right)$and $q^{+}=q^{*}\left(p^{+}\right)$; then $q^{-}<q^{+}$as well. Note that, by symmetry, $\Phi_{-i, j-1}(x)=(N-1) q^{-}<(N-1) q^{+}=\Phi_{-i, j}(x)$. For $p<0$ we have

$$
\begin{aligned}
& \min \left\{H_{i, j-1}(x, p), H_{i, j}(x, p)\right\} \\
& =\frac{\sigma}{1-\sigma}(-p)^{1-1 / \sigma}+p\left(\max \left\{\Phi_{-i, j-1}(x), \Phi_{-i, j}(x)\right\}-g(x)\right)=H_{i, j}(x, p),
\end{aligned}
$$

and hence

$$
F_{i, j}^{*}(x, W(x), p)=\rho W(x)-H_{i, j}(x, p) .
$$

As $H_{i, j-1}(x, p) \geq H_{i, j}(x, p)$, Equation (33) implies

$$
0 \leq \rho W(x)-H_{i, j}\left(x, p^{-}\right)=F_{i, j}^{*}\left(x, W(x), p^{-}\right)
$$

as well as

$$
0=F_{i, j}^{*}\left(x, W(x), p^{+}\right) .
$$

As $F_{i, j}^{*}(x, W(x), p)$ is a concave function of $p$ that is nonnegative on the endpoints of the interval $D^{-} W(x)=\left[p^{-}, p^{+}\right]$, it is nonnegative for all points in the interval, and $W$ is a supersolution at $x$.

The second situation to be investigated is $p^{-}>p^{+}$. In that case $D^{+} W(x)=$ $\left[p^{+}, p^{-}\right]$and $D^{-} W(x)=\emptyset$. Now $W$ is automatically a supersolution at $x$, and we have to check whether it is a subsolution.

Let, as before, $q^{-}=q^{*}\left(p^{-}\right)$and $q^{+}=q^{*}\left(p^{+}\right)$; then $q^{-}>q^{+}$and $\Phi_{-i, j-1}(x)>$ 
$\Phi_{-i, j}(x)$. In this case we have for $p<0$

$$
\begin{aligned}
& \max \left\{H_{i, j-1}(x, p), H_{i, j}(x, p)\right\} \\
& =\frac{\sigma}{1-\sigma}(-p)^{1-1 / \sigma}+p\left(\min \left\{\Phi_{-i, j-1}(x), \Phi_{-i, j}(x)\right\}-g(x)\right)=H_{i, j}(x, p)
\end{aligned}
$$

We have again (33), and therefore $q^{-}>a_{1}(x)>d(x)$, and, by the second jump condition, also $q^{+} \geq d(x)$. Therefore

$$
\frac{\partial H_{i, j}}{\partial p}\left(x, p^{+}\right)=N q^{+}-g(x) \geq 0,
$$

and, by convexity of $H_{i, j}(x, p)$, we have that $H_{i, j}(x, p) \geq H_{i, j}\left(x, p^{+}\right)$for all $p>p^{+}$. This implies

$$
F_{* i, j}(x, W(x), p) \leq F_{* i, j}\left(x, W(x), p^{+}\right)
$$

for all $p \in D^{+} W(x)$, whence we conclude that $W(x)$ is also a subsolution at $x$.

\section{A.12 Proof of Theorem 4.18}

Proof. The 'only if' part follows directly from Propositions 4.14 and 4.15.

To see the 'if', note that any point in $\left(\tilde{x}_{0}, \tilde{q}_{0}\right) \in \Xi$ can be extended both left and right to form a fully specified $\phi(x)$. The extension is undertaken as follows: follow the orbit of the shadow price system left from $\tilde{x}_{0}$ until the first discontinuity at $\left(\tilde{x}_{-1}, \tilde{q}_{-1}^{+}\right)$. At the point of the discontinuity, the strategy jumps to $\left(\tilde{x}_{-1}, \tilde{q}_{-1}^{-}\right)$. By Lemma 5.1, this point is uniquely defined and lies on the opposite side of $A_{1}$. Keep extending left until the next discontinuity at $\left(\tilde{x}_{-2}, \tilde{q}_{-2}^{+}\right)$, and so on for all the discontinuities in $j \in\left\{1, \ldots, J^{-}\right\}$, with $J^{-} \geq 0$. A discontinuity must be placed at a point at which the present orbit of the shadow price system would exit $\Xi$; such points include all points $(x, d(x))$ for $x \leq \hat{x}$ and all points $\left(x, \Phi^{s \dagger}(x)\right)$ for $x>\hat{x}$. A discontinuity cannot be placed where the extension of the orbit left from $\left(\tilde{x}_{-j}, \tilde{q}_{-j}^{-}\right)$would immediately exit $\Xi$; such points include all points $\left(x, \Phi^{s}(x)\right)$ for $x>\hat{x}$ and all points $\left(x, d^{\dagger}(x)\right)$ for $x \leq \check{x}$.

Extending to the right takes places symmerically, with discontinuities (if any) between $\left(\tilde{x}_{j}, \tilde{q}_{-j}^{-}\right)$and $\left(\tilde{x}_{j}, \tilde{q}_{-j}^{+}\right)$for $j \in\left\{1, \ldots, J^{+}\right\}$with $J^{+} \geq 0$. A discontinuity must be inserted where the orbit of the shadow price system extending to the right from $\left(\tilde{x}_{j}, \tilde{q}_{-j}^{-}\right)$exits $\Xi$ immediately; these points are given by all points $\left(x, d^{\dagger}(x)\right)$ for $x \geq \hat{x}^{*}$ and all points $\left(x, \Phi^{s \dagger}(x)\right)$ for $x<\bar{x}$. A discontinuity cannot be inserted where the right extension of $\left(\tilde{x}_{j}, \tilde{q}_{-j}^{+}\right)$exits $\Xi$ immediately; these points are given by all points $\left(x, \Phi^{s}(x)\right)$ for $x<\bar{x}$ and by all points $(x, d(x))$ for $x \geq \hat{x}$. 


\section{A.13 Proof of Lemma 5.1}

Proof. By Lemma 4.1, for any $x, G(x, p)$ has a critical point where $h_{1}^{*}(x, p)=0$. As $p^{*}(q)$ is invertible, $\tilde{G}(x, q)=G\left(x, p^{*}(q)\right)$ takes a global minimum at $q=a_{1}(x)$. Hence $\tilde{G}(x, q)$ is decreasing for $m \leq q \leq a_{1}(x)$ and increasing for $a_{1}(x) \leq q \leq M$. Since

$$
V_{i}(x)=\frac{1}{\rho} \tilde{G}\left(x, \phi_{i}(x)\right), \quad i=1,2,
$$

by equation (11), the result follows.

\section{A.14 Proof of Theorem 5.2}

Proof. Proposition 4.15 rules out any equilibrium strategy lying in the interior of the convex hull of $\Phi^{s}(x)$ and $\Phi^{s \dagger}(x)$. The former is the continuous strategy, and by definition the latter yields the same value. By Lemma 5.1, any equilibrium strategy which yields a lower value would have to lie in the interior of the convex hull.

\section{References}

Achdou, Y., Buera, F.J., Lasry, J.M., Lions, P.L., Moll, B., 2014. Partial differential equation models in macroeconomics. Philosophical Transactions of the Royal Society A: Mathematical, Physical and Engineering Sciences 372, 20130397.

Achdou, Y., Han, J., Lasry, J.M., Lions, P.L., Moll, B., 2017. Income and wealth distribution in macroeconomics: A continuous-time approach. Technical Report 23732. National Bureau of Economic Research.

Akao, K.I., Uchida, K., Wasa, Y., 2018. International environmental agreements as an equilibrium choice in a differential game. mimeo.

Alós-Ferrer, C., Ritzberger, K., 2008. Trees and extensive forms. Journal of Economic Theory 143, 216-250.

Bardi, M., Capuzzo-Dolcetta, I., 2008. Optimal control and viscosity solutions of Hamilton-Jacobi-Bellman equations. Birkhäuser Boston.

Barles, G., Briani, A., Chasseigne, E., 2013. A Bellman approach for twodomains optimal control problems in $\mathbb{R}^{N}$. ESAIM: Control, Optimisation and Calculus of Variations 19, 710-739.

Barles, G., Briani, A., Chasseigne, E., 2014. A Bellman Approach for Regional Optimal Control Problems in $\mathbb{R}^{N}$. SIAM Journal on Control and Optimization $52,1712-1744$.

Başar, T., Olsder, G.J., 1999. Dynamic Noncooperative Game Theory. second ed., SIAM, Philadelphia, PA. 
Battaglini, M., Nunnari, S., Palfrey, T.R., 2014. Dynamic free riding with irreversible investments. American Economic Review 104, 2858-71.

Battaglini, M., Nunnari, S., Palfrey, T.R., 2016. The dynamic free rider problem: A laboratory study. American Economic Journal: Microeconomics 8, 268-308.

Bernheim, B.D., Ray, D., 1989. Collective dynamic consistency in repeated games. Games and Economic Behavior 1, 295-326.

Bressan, A., 2009. From optimal control to non-cooperative differential games: a homotopy approach. Control and Cybernetics 38, 1081-1106.

Clarke, F., 2004. Lyapunov functions and feedback in nonlinear control, in: Optimal control, stabilization and nonsmooth analysis. Springer, pp. 267-282.

Cvitanić, J., Georgiadis, G., 2016. Achieving efficiency in dynamic contribution games. American Economic Journal: Microeconomics 8, 309-42.

De Frutos, J., Martín-Herrán, G., 2018. Selection of a markov perfect nash equilibrium in a class of differential games. Dynamic Games and Applications $8,620-636$.

Dockner, E., Sorger, G., 1996. Existence and properties of equilibria for a dynamic game on productivity assets. Journal of Economic Theory 71, 209-227.

Dockner, E., Wagener, F., 2014. Markov perfect Nash equilibria in models with a single capital stock. Economic Theory 56, 585-625.

Dockner, E.J., Jørgensen, S., van Long, N., Sorger, G., 2000. Differential Games in Economics and Management Science. CUP, Cambridge, UK.

Dockner, E.J., Long, N.V., 1993. International pollution control: cooperative versus noncooperative strategies. Journal of Environmental Economics and Management 25, 13-29.

Dutta, P.K., Sundaram, R.K., 1993. The tragedy of the commons? Economic Theory 3, 413-426.

Ekeland, I., Karp, L., Sumaila, R., 2015. Equilibrium resource management with altruistic overlapping generations. Journal of Environmental Economics and Management 70, 1-16.

Farrell, J., Maskin, E., 1989. Renegotiation in repeated games. Games and economic behavior 1, 327-360.

Fershtman, C., Nitzan, S., 1991. Dynamic voluntary provision of public goods. European Economic Review 35, 1057-1067.

Filippov, A.F., 1988. Differential Equations with Discontinuous Righthand Sides. Springer. 
Fleming, W.H., Soner, H.M., 2006. Controlled Markov processes and viscosity solutions. Springer, New York.

Fudenberg, D., Tirole, J., 1991. Game Theory. MIT Press, Cambridge, MA.

Georgiadis, G., 2015. Projects and team dynamics. The Review of Economic Studies 82, 187-218.

Harstad, B., 2012. Climate contracts: A game of emissions, investments, negotiations, and renegotiations. Review of Economic Studies 79, 1527-1557.

Hirsch, M., Pugh, C., Shub, M., 1977. Invariant manifolds. volume 583 of Lecture Notes in Mathematics. Springer, Heidelberg.

Klein, N., Rady, S., 2011. Negatively correlated bandits. The Review of Economic Studies 78, 693-732.

Levhari, D., Mirman, L.J., 1980. The great fish war: an example using a dynamic cournot-nash solution. The Bell Journal of Economics , 322-334.

Long, N.V., 2011. Dynamic games in the economics of natural resources: a survey. Dynamic Games and Applications 1, 115-148.

Long, N.V., 2012. Applications of dynamic games to global and transboundary environmental issues: a review of the literature. Strategic Behavior and the Environment 2, 1-59.

Maskin, E., Tirole, J., 2001. Markov perfect equilibrium: I. Observable actions. JET 100, 191-219.

Nuño, G., Moll, B., 2018. Social optima in economies with heterogeneous agents. Review of Economic Dynamics 28, 150-180.

Rincón-Zapatero, J., Martínez, J., Martín-Herrán, G., 1998. New method to characterize subgame perfect Nash equilibria in differential games. Journal of Optimization Theory and Applications 96, 377-395.

Rowat, C., 2007. Non-linear strategies in a linear quadratic differential game. Journal of Economic Dynamics and Control 31, 3179-3202.

Sorger, G., 1998. Markov-perfect Nash equilibria in a class of resource games. Economic Theory 11, 79-100.

Tsutsui, S., Mino, K., 1990. Nonlinear strategies in dynamic duopolistic competition with sticky prices. Journal of Economic Theory 52, 136-161.

Vespa, E., 2020. An experimental investigation of cooperation in the dynamic common pool game. International Economic Review 61, 417-440.

Wagener, F., 2003. Skiba points and heteroclinic bifurcations, with applications to the shallow lake system. Journal of Economic Dynamics and Control 27, $1533-1561$. 
Wirl, F., 2007. Do multiple Nash equilibria in Markov strategies mitigate the tragedy of the commons? Journal of Economic Dynamics and Control 31, $3723-3740$.

Wirl, F., 2014. Uniqueness versus indeterminacy in the tragedy of the commons: A 'geometric' approach, in: Dynamic Optimization in Environmental Economics. Springer, pp. 169-192. 